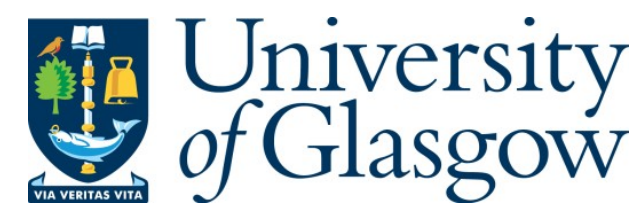

Sugimoto, Y ., Radice, G., Ceriotti, M ., and Sanchez, J.P. (2014) H azardous near Earth asteroid mitigation campaign planning based on uncertain information on fundamental asteroid characteristics. A cta A stronautica, 103. pp. 333-357. ISSN 0094-5765

Copyright $\odot 2014$ Elsevier

A copy can be downloaded for personal non-commercial research or study, without prior permission or charge

Content must not be changed in any way or reproduced in any format or medium without the formal permission of the copyright holder(s)

When referring to this work, full bibliographic details must be given

http://eprints.gla.ac.uk/92489/

Deposited on: 20 M arch 2014

Enlighten - Research publications by members of the University of Glasgow http://eprints.gla.ac.uk 


\title{
Hazardous near Earth asteroid mitigation campaign planning based on uncertain information on
}

\section{fundamental asteroid characteristics}

\author{
Y. Sugimoto $^{(1)}$, G. Radice ${ }^{(2)}$, M. Ceriotti ${ }^{(3)}$, and J. P. Sanchez ${ }^{(4)}$ \\ ${ }^{(1)}$ University of Glasgow, Glasgow G12 8QQ UK, +44 (0)141 330 8470, y.sugimoto.1@ research.gla.ac.uk \\ ${ }^{(2)}$ University of Glasgow, Glasgow G12 8QQ UK, +44 (0)141330 6465, matteo.ceriotti@glasgow.ac.uk \\ ${ }^{(3)}$ University of Glasgow, Glasgow G12 8QQ UK, +44 (0)141 330 4068, gianmarco.radice@glasgow.ac.uk \\ ${ }^{(4)}$ Universitat Politècnica de Catalunya, Calle Jordi Girona, 31, 08034 Barcelona Spain, +34 (0)934 013493 , \\ jpau.sanchez@upc.edu
}

\begin{abstract}
Given a limited warning time, an asteroid impact mitigation campaign would hinge on uncertainty-based information consisting of remote observational data of the identified Earth-threatening object, general knowledge of near-Earth asteroids (NEAs), and engineering judgment. Due to these ambiguities, the campaign credibility could be profoundly compromised. It is therefore imperative to comprehensively evaluate the inherent uncertainty in deflection and plan the campaign accordingly to ensure successful mitigation. This research demonstrates dual-deflection mitigation campaigns consisting of primary (instantaneous/quasi-instantaneous) and secondary (slow-push) deflection missions, where both deflection efficiency and campaign credibility are taken into account. The results of the dual-deflection campaign analysis show that there are trade-offs between the competing aspects: the launch cost, mission duration, deflection distance, and the confidence in successful deflection. The design approach is found to be useful for multi-deflection campaign planning, allowing us to select the best possible combination of missions from a catalogue of campaign options, without compromising the campaign credibility.
\end{abstract}

Keywords: near-Earth asteroid, deflection technique, dual-deflection campaign, uncertain information, multi-objective optimization

\section{Nomenclatures}

\begin{tabular}{|c|c|c|}
\hline $\mathbf{p}$ & $:$ & vector of mitigation system design parameters \\
\hline $\mathbf{x}$ & $:$ & vector of mitigation system design variables \\
\hline $\mathbf{y}$ & : & vector of mitigation system indicators \\
\hline$\beta$ & : & momentum multiplication/enhancement factor \\
\hline$M_{a}$ & : & asteroid mass, $\mathrm{kg}$ \\
\hline$p_{v}$ & : & geometric albedo value \\
\hline$\rho_{\text {micro }}$ & : & micro-density, $\mathrm{g} / \mathrm{cm}^{3}$ \\
\hline$P_{\text {bulk }}$ & : & bulk-porosity \\
\hline$d$ & : & equivalent diameter of asteroid, $\mathrm{m}$ \\
\hline $\mathbf{v}_{\infty}$ & $:$ & unperturbed geocentric hyperbolic excess velocity vector \\
\hline b & : & impact parameter/b-plane deflection vector \\
\hline $\mathrm{R}_{\oplus}$ & : & Earth's radius, $6,371 \mathrm{~km}$ \\
\hline$b_{\oplus}$ & : & b-plane deflection scaling actual deflection equal to the Earth's radius $\mathrm{R}_{\oplus}$ \\
\hline $\mathrm{v}_{\mathrm{e}}$ & : & Earth's escape velocity, $11.2 \mathrm{~km} / \mathrm{s}$ \\
\hline$v_{\infty}$ & : & $\left\|\mathbf{v}_{\infty}\right\|$ \\
\hline G & : & gravity constant, $6.67259 \times 10^{-11} \mathrm{~m}^{3} / \mathrm{kg} / \mathrm{s}^{2}$ \\
\hline $\mathrm{M}_{\oplus}$ & : & mass of the Earth, $5.9742 \times 10^{24} \mathrm{~kg}$ \\
\hline $\mathrm{M}_{\odot}$ & : & mass of the Sun, $1.98855 \times 10^{30} \mathrm{~kg}$ \\
\hline
\end{tabular}




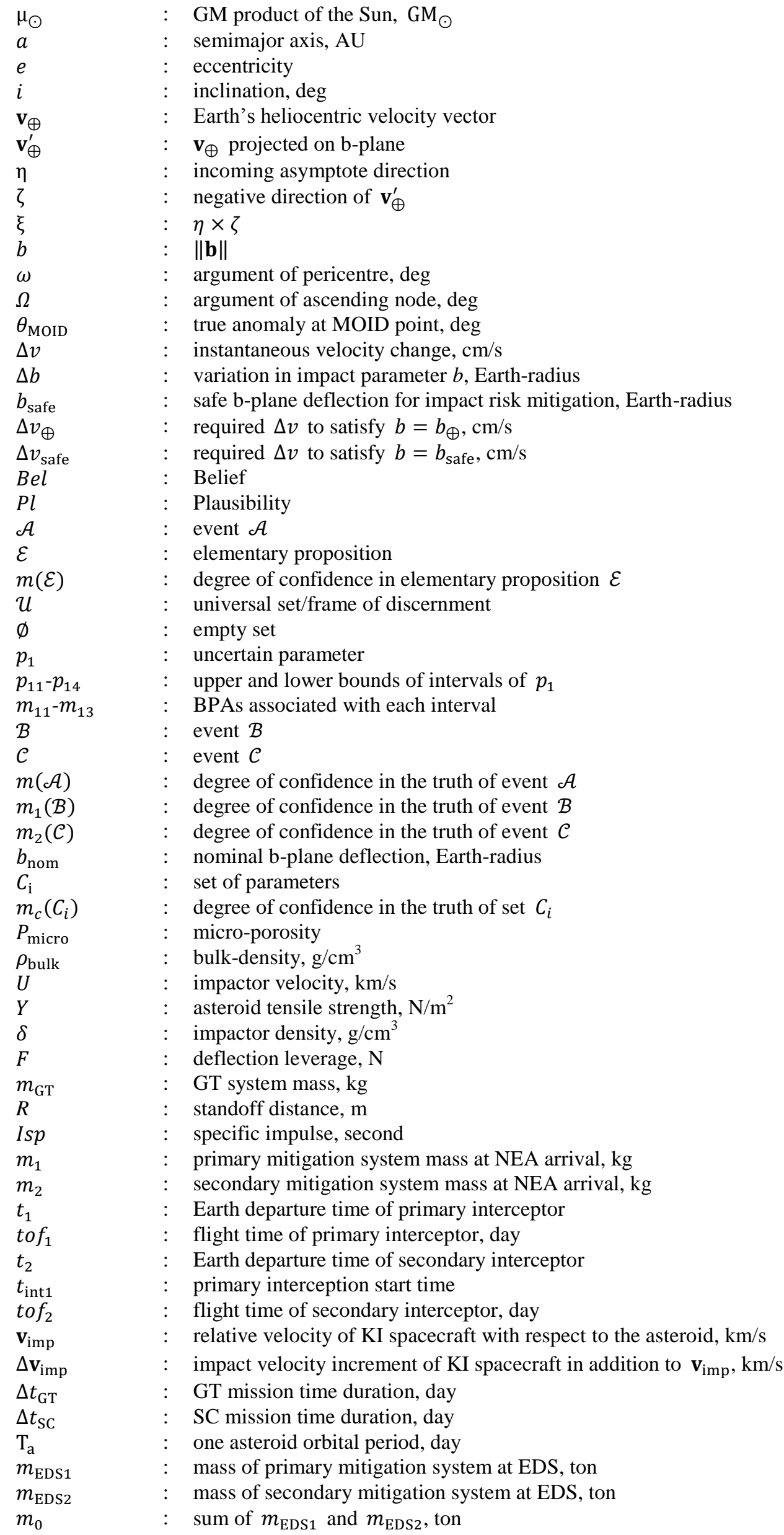




$\begin{array}{ll}t_{\mathrm{f} 1} & : \text { asteroid arrival time of primary mitigation system } \\ t_{\mathrm{f} 2} & : \text { asteroid arrival time of secondary mitigation system } \\ B e l_{\mathrm{nom}} & : \text { Pelief of } b_{\mathrm{nom}} \\ P l_{\mathrm{nom}} & : \text { plausibility of } b_{\text {nom }} \\ b_{\text {trim }} & : \text { KI system mass } \\ m_{\mathrm{KI}} & : \text { fraction of } m_{\mathrm{KI}} \text { that makes an impact with the asteroid } \\ m_{\mathrm{imp}} & : \text { propellant mass for KI system's terminal guidance } \\ \left.m_{\mathrm{prop}}\right|_{\mathrm{TG}} & : \text { NI system mass } \\ m_{\mathrm{NI}} & : \text { nuclear warhead mass } \\ m_{\mathrm{WH}} & : \text { SC system mass } \\ m_{\mathrm{SC}} & : \text { collector assembly mass } \\ m_{\mathrm{CA}} & : \text { diameter of collector } \\ d_{\mathrm{SC}} & : \text { GT system mass } \\ m_{\mathrm{GT}} & : \text { dry mass } \\ m_{\mathrm{dry}} & : \text { propellant mass for hovering } \\ \left.m_{\mathrm{prop}}\right|_{\text {hover }} & \text { power supply mass } \\ m_{\mathrm{ps}} & \end{array}$

\section{Introduction}

As of today, several asteroid deflection concepts have been proposed and they are under preliminary investigation. Some of these concepts appear to be feasible with the current technology developed through deep space exploration missions, whereas others require certain levels of technological advancement before they can be considered as feasible deflection alternatives. Also, a deflection technique which makes use of nuclear devices for example, involves political issues to be tackled with in global cooperation. Nevertheless, we now recognise that it is not unrealistic to prevent an impact event by a modest-sized ( $<150$ metres in diameter) near-Earth asteroid (NEA) if it can be discovered and identified to be threatening about a decade in advance of the impact event [1]. Most importantly, even such small asteroids can cause a local devastation far greater than the Tunguska event in 1908 or the Chelyabinsk meteor event on the 15th of February in 2013. Fortunately, statistically speaking, or based on the NEA population that has been discovered so far, it is more likely that hazardous NEAs to be mitigated in the foreseeable future will be in this modest size range, rather than kilometre-sized NEAs which can potentially trigger a global catastrophe such as the Cretaceous-Palaeogene (K-Pg) extinction event [2]. Although it is not obvious that a kilometre-sized object will not collide with the Earth in the near future as those undiscovered may be on collisional trajectory, the size of an Earth-threatening object basically scales the cost of mitigation campaigns (of course, this is not always the case if the use of high yield-to-weight nuclear interception is allowed for instance) but is not significant to the main focuses of this study: the uncertain information and mitigation campaign credibility. While characterisation of larger NEAs may be done more accurately and affect mitigation mission design, this paper does not intend to focus on kilometre-sized objects but it will only deal with hazard mitigation of the modest, 140-metre sized NEAs with warning time of 10-20 years for conciseness. This range of warning time is rather optimistic for hundred-metre sized threatening objects, considering the limited capability of asteroid orbit determination by today's telescopic surveillance [3]. Note that the warning time in this paper is defined as the time period between the detection of a hazardous NEA and the Earth impact epoch of the hazardous NEA; however, it does not take the mission preparation time into consideration. It is assumed that mitigation systems will be developed and fabricated based on a spacecraft bus in a similar way to the interceptor stack concept proposed by Adams et al. [4] such that the preparation time does not require many years unlike conventional space exploration missions. Readers 
interested in more detailed analysis on Technology Readiness Levels (TRLs) of different mitigation systems should refer to the existing literature by Sanchez et al. [1].

The most notable feature of asteroid deflection mission is that the characteristics (orbital parameters, physical properties, dynamical properties, etc.) of the NEA are deeply embedded into the design as an integral part of the mitigation systems, and influence their deflection performance. Figure 1 is a schematic diagram that describes such hazardous asteroid mitigation system design as multidisciplinary system design. The mitigation system design involves three basic vectors $\mathbf{p}, \mathbf{x}$, and $\mathbf{y}$ where

- $\mathbf{p}$ is a vector of design parameters representing fundamental properties of the hazardous NEA (e.g. orbit, physical property, etc.) and environmental parameters (e.g. gravity, solar constant, radiation pressure, etc.).

- $\mathbf{x}$ is a vector of mitigation system design variables (e.g. mass and impact velocity of kinetic impactor (KI), mass of nuclear interceptor (NI), mirror size of solar collector (SC), mass and hovering altitude of gravity tractor (GT), etc.).

- $\mathbf{y}$ is a vector of mitigation performance indicators of campaign (e.g. launch cost, mission duration, deflection distance, confidence in successful deflection, etc.).

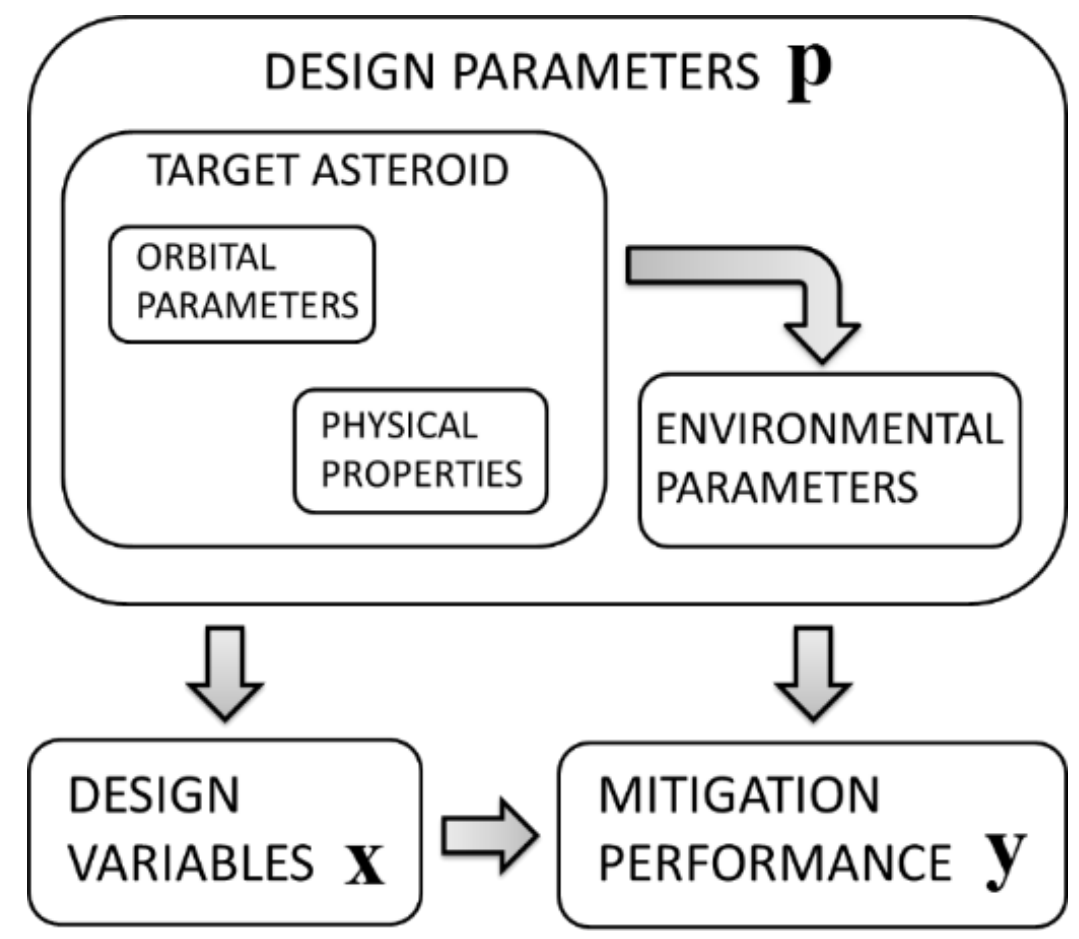

Figure 1 Hazardous NEA mitigation system design.

Recent work by Sugimoto et al. [5] has shown that, for the 10-year warning time impact scenarios, only limited information about the hazardous NEA would be available and that this will most likely come only from ground-based or space-based characterisation approaches. In such cases, the majority of deflection techniques will be subject to epistemic uncertainties and measurement errors in the NEA characteristics, which could lead to compromised outcomes of mitigation. It is therefore essential to investigate mitigation campaign planning 
that involves design of a reliable and robust hazardous NEA mitigation system. The mitigation campaign should guarantee high confidence in successful mitigation even if the preliminary NEA characterisation is incomplete.

The main objective of this research is to demonstrate a mitigation campaign planning approach that results in efficient, reliable, and yet robust hazardous NEA mitigation for the 10-20 warning-time scenarios. The additional objective is to ensure the flexibility in deflection in order to avoid undesired key-hole passage on the b-plane [6] due to the primary deflection.

To fulfil these objectives, we have considered a dual-deflection mitigation approach that makes use of an instantaneous/quasi-instantaneous deflection technique (KI/NI/SC) as a primary deflection mission and a slow-push deflection technique (GT) as a secondary deflection mission. The use of a GT as a secondary deflection mission for the secondary impact keyhole avoidance was suggested in the JPL report by Yeomans et al. [7] in 2008. They also pointed out that tracking of the GT spacecraft would provide precise information about the asteroid orbit before and after the primary deflection mission and also after the GT trim manoeuvre. Their study however, assumed the range of the momentum enhancement factor $\beta$ of the NEA $(1<\beta<5)$ in order to evaluate possible outcomes of the primary deflection achieved through a KI mission instead of considering the uncertainties in the NEA characteristics. Such combined mitigation measures have been also investigated as a part of the NEOShield project [8].

Design of a dual-deflection mitigation campaign involves trade-offs between the competing aspects (the launch cost, mission duration, deflection distance, and the confidence in successful deflection) which are to be optimised in order to minimise the launch cost of hazardous NEA mitigation systems and total campaign period while maximising the deflection performance and the confidence in successful mitigation campaign.

This paper is articulated as follows. In Section 2, the fundamental aspects of preliminary NEA characterisation subject to the available warning time, associated epistemic uncertainties, and inevitable measurement errors are briefly highlighted. Section 3 gives an overview of hazardous NEA deflection missions of KI, NI, SC, and GT and associated uncertainty in the outcomes of deflection missions due to the incomplete information on the characteristics of the target NEA. Section 4 details the planning of a combined hazardous NEA mitigation campaign consisting of two different deflection missions, followed by the mitigation campaign optimisation approach used in this paper. Finally, we present the results of analysis on the dual-deflection campaigns consisting of a KI/NI/SC mission backed up by a GT mission in Section 5.

\section{Preliminary characterisation of hazardous NEA}

Preliminary characterisation of an identified hazardous NEA is essential during the early stages of mitigation campaign planning to appropriately design mitigation systems based on the available information regarding the fundamental characteristics (e.g. physical, dynamical, orbital properties, etc.) of the target NEA. Sending a precursor mission to the NEA is obviously preferable in terms of measurement accuracy as well as to avoid possible mischaracterisation in size, mass, etc.; however, in reality, available characterisation options will be dependent on the given warning time and the NEA orbit.

In the following subsections, three different characterisation approaches - ground-based, space-based, and proximity characterisation - are presented and their degrees of measurement accuracy are briefly summarised. The ground-based characterisation approach is explained more in detail here along with its characterisation diagram as this is the most likely characterisation scenario. Two types of uncertainties - epistemic uncertainties and measurement errors - associated with the preliminary NEA characterisation are then introduced. In addition, 
aleatory uncertainties are presented for reference, since these are related to the practical limitations (i.e. errors) on mitigation systems (e.g. lack of precision).

\subsection{Characterisation scenarios}

Depending on the available warning time, preliminary characterisation of a hazardous NEA will vary since each characterisation approach will have a different degree of uncertainty. There are basically three different preliminary characterisation approaches: ground-based, space-based, and proximity characterisation.

The ground-based characterisation makes use of telescopic and radar observations from the Earth whereas the space-based characterisation leverages infrared astronomical satellites (IRAS) in space in addition to the ground-based observation options. These two characterisation scenarios would require only a few days during close Earth approaches of NEAs to complete the preliminary characterisation [9], which means they could be possibly used simultaneously with the first discovery of a hazardous NEA by radar or telescope. On the other hand, the proximity characterisation approach, which requires a precursor mission to the target NEA, would take $<1 \frac{1}{4}$ years to complete the preliminary characterisation [10]. The availability of precursor mission is subject to the orbit of an identified threatening NEA while, particularly for the 10-20 warning-time cases, quick preliminary characterisation is essential to ensure a wider mitigation campaign window (i.e. the period between the Earth departure of mitigation systems and the completion of NEA mitigation campaign). The wider campaign window results in more mitigation campaign options and more efficient mitigation, even though the preliminary characterisation may remain incomplete without a precursor mission.

The accuracy of observational information by the ground-based characterisation is based on the capability of ground-based telescopes and radar instruments on Earth, and thus the majority of physical parameters of the target object will remain highly uncertain [11]. Microscopic properties of a NEA can be estimated by analysing the surface colour and solar spectral reflectance while macroscopic characteristics such as the mass and the porosity are much more difficult to be ascertained particularly when the object is only a few hundred metres or so in diameter. According to Müller et al. [12], the ground-based telescopic observations with the Thermal Infrared Multimode Instrument dedicated to 3-25 micron range (TIMMI2) have demonstrated higher performance at NEA characterisation than the radar telescopic observations (e.g. Arecibo and Goldstone) as the asteroid sub-surface/internal structures can be roughly estimated through thermal characterisation of the asteroidal surface. However, in this study, such advanced ground-based mid-infrared observations are not considered to distinguish the ground-based characterisation from the space-based characterisation which makes use of infrared observation.

Figure 2 is the simplified diagram of the ground-based characterisation approach. The fundamental physical characteristics (mass, size, albedo, and momentum enhancement) for mitigation system design are derived from the ground-based observational data, meteorite analogues of the NEA, and expert opinions regarding the macro porosity (i.e. large structural flaws inside the NEA). Crucially, the mass determination of the NEA cannot be done directly through the ground-based observations since it requires additional information from meteorite analogues regarding the microscopic characteristics of the main material that composes the asteroid and expert opinions regarding the macro porosity to estimate the bulk density of the NEA. It is therefore inevitable that there would be major uncertainties in the preliminary characterisation, particularly in the mass (i.e. bulk density) and albedo value for the case of ground-based characterisation. 


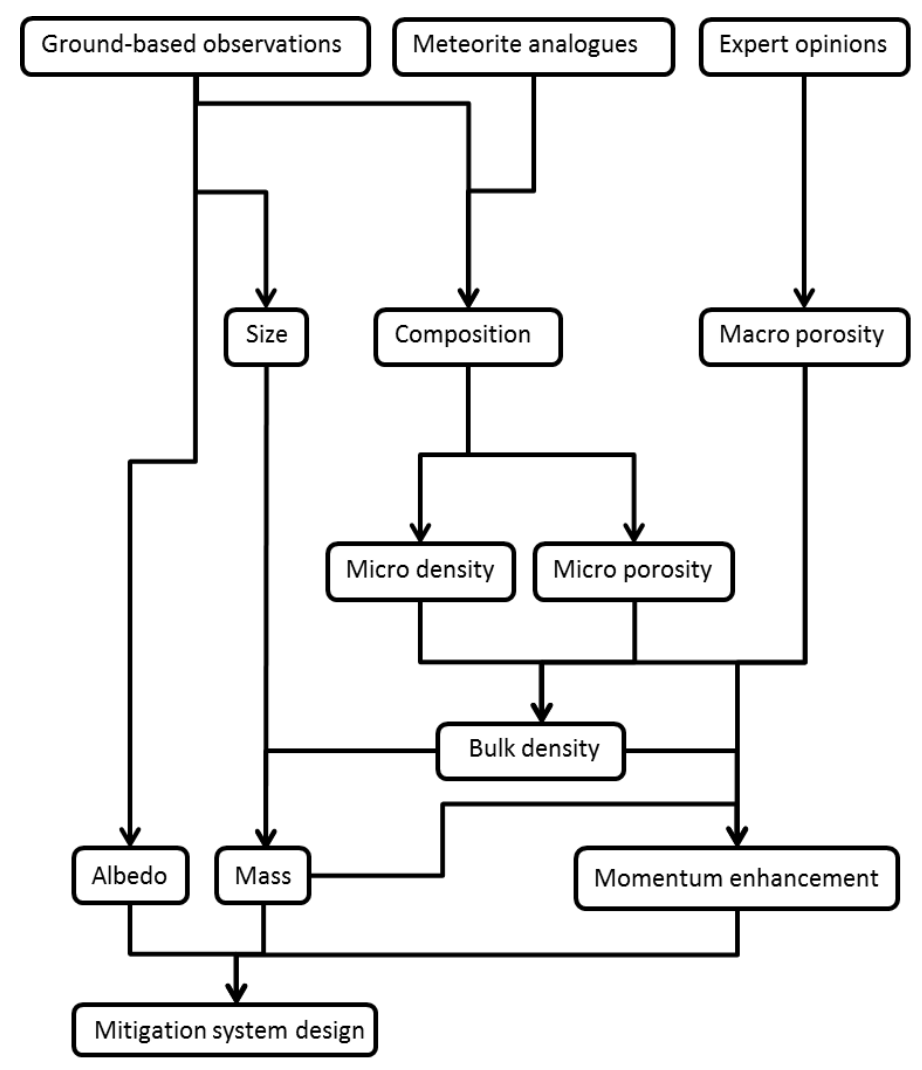

Figure 2 Ground-based characterisation diagram.

\subsection{Types of uncertainty}

There are three basic types of uncertainty - epistemic uncertainty, error (i.e. numerical uncertainty), and aleatory uncertainty - derived from different sources regarding the NEA characteristics or mitigation systems.

In general, epistemic uncertainties arise when a system is not sufficiently characterised, certain characteristics of the system are neglected, or the physical model of the system is based on hypotheses rather than experiments. Our current characterisation on the NEA population including potentially hazardous asteroids is insufficient, hence epistemic uncertainties exist in general knowledge of NEAs. This type of uncertainties can be reduced by obtaining more credible information on the specific threatening NEAs or by improving general knowledge of the NEA population. Moreover, epistemic uncertainties also exist in design of low TRL components of a mitigation system such as the solar-pumped laser ablation system [13]. Unlike aleatory uncertainties, representation of epistemic uncertainties requires an appropriate quantification technique such as Evidence Theory [14].

Errors (i.e. numerical uncertainties) are a recognisable deficiency due to practical constraints on a system in general. The in-situ mass determination by the Hayabusa spacecraft, for example, had about $5 \%$ of measurement error which is thought to be due to the relatively small mass of Itokawa [15] - 190,000 times smaller than that of Eros, whose mass was determined within $0.05 \%$ [16]. The size determination of NEAs from the ground is also subject to the resolution of telescopic and radar images due to the practical constraints on the ground-based observational instruments. This type of uncertainties can be minimised by simply applying more accurate measurement techniques. Unlike the other types of uncertainties, the source is often known such that one can often estimate error magnitudes in advance. Measurement errors in NEA size, rotation state, and shape determination could be as significant as epistemic uncertainties in mass determination, however the hazardous 
asteroid is modelled as a spherical body throughout this work and these problems are not dealt with. Care must be therefore taken for the accuracy/applicability of the results in this paper by the readers intending to compare their study on asteroid deflection techniques applied to a more realistic misshapen asteroid model for instance. Brief discussion on how the asteroid shape affects the deflection techniques is provided in subsection 3.1.

Aleatory uncertainties (also known as inherent uncertainties) represent a random variation in a system, which is inevitably present in every outcome of the system. For the case of KI, there will be, for instance, a certain amount of possibility of missing the target point on the NEA surface, or at worst the NEA itself due to aleatory uncertainties in the precision of the KI system. Such practical limitations of the KI technique is associated with epistemic uncertainties and measurement errors in NEA size, rotation state, shape, centre of gravity, etc., and a very high-velocity $(10-20 \mathrm{~km} / \mathrm{s}$ or $>30 \mathrm{~km} / \mathrm{s}$ for retrograde orbits) impact relative to a modest-sized object in space. For the case of SC, the acceleration of the target body (i.e. the surface material evaporation rate) will always fluctuate due to aleatory uncertainties in the solar flux, the asteroid surface condition, the mirror degradation with time. This type of uncertainties cannot be completely eliminated but they can be mathematically modelled using a conventional probability theory, once a sufficient amount of statistical data is available. For example, the effects of aleatory uncertainties on asteroid deflection actions can be evaluated by means of Monte-Carlo simulation; however, applying a Monte-Carlo simulation to the mitigation campaign planning and optimisation approach considered in this study will substantially increase the cost of computation. The authors are currently working on asteroid deflection mission analysis on this aspect, the results of which are excluded from this paper. Care must be therefore taken when dealing with the results of this paper and comparing with the results from other literature that take the aleatory uncertainties into account.

Epistemic uncertainties will be the most common cause of the uncertain physical properties of the target body, followed by measurement errors unless our general knowledge of NEAs is abundant and unbiased. Table 1 summarises the uncertainties in NEA mass $M_{a}$ and geometric albedo $p_{v}$ corresponding to the three different characterisation options and three different taxonomic classes: S-type, C-type, and M-type, where the NEA mass $M_{a}$ can be determined by referring to the micro density $\rho_{\text {micro }}$, bulk porosity $P_{\text {bulk }}$, and the equivalent diameter $d$ of the NEA, according to Equation (1).

$$
M_{a}=\rho_{\text {micro }}\left(1-P_{\text {bulk }}\right) \times 4 \pi(d / 2)^{3} / 3
$$

These uncertainties originate from the lower and upper bounds of the uncertain NEA physical properties given in Table 12 in Appendix A.1. As can be seen, more rigorous but possibly more time-consuming characterisation results in smaller ranges of uncertainties in the physical properties for all the taxonomic classes. Interested readers should refer to Appendix A.1 for further details on the uncertain NEA physical properties. 
Table 1 Uncertainties in the mass $M_{a}$ and the geometric albedo $p_{v}$ of the NEA corresponding to the ground-based, space-based, and the proximity characterisation scenarios. They are represented in percentage relative to the mean physical properties of each taxonomic class. A) S-type. B) C-type. C) M-type.

\begin{tabular}{cccc}
\hline A) & Ground-based & Space-based & Proximity \\
\hline$M_{a}$ & $-36.1-45.7 \%$ & $-28.8-36.8 \%$ & $-5.4-9.0 \%$ \\
$p_{v}$ & $-46.4-50.0 \%$ & $-23.8-31.7 \%$ & $-10.0-10.0 \%$ \\
\hline B) & Ground-based & Space-based & Proximity \\
\hline$M_{a}$ & $-41.3-45.6 \%$ & $-32.3-35.5 \%$ & $-4.0-4.7 \%$ \\
$p_{v}$ & $-26.5-34.1 \%$ & $-23.8-31.7 \%$ & $-10.0-10.0 \%$ \\
\hline C) & Ground-based & Space-based & Proximity \\
\hline$M_{a}$ & $-27.3-24.5 \%$ & $-21.5-20.8 \%$ & $-3.0-2.0 \%$ \\
$p_{v}$ & $-28.6-42.9 \%$ & $-23.8-31.7 \%$ & $-10.0-10.0 \%$ \\
\hline
\end{tabular}

\section{Hazardous NEA deflection}

The deflection representation of hazardous NEAs in this work is based on the b-plane concept that is applied to planetary encounter analyses [6]. The b-plane is oriented normal to the incoming asymptote of the osculating geocentric hyperbola, in other words, it is oriented normal to the object's unperturbed geocentric/hyperbolic excess velocity vector $\mathbf{v}_{\infty}$ as shown in Figure 3. The b-plane analysis can not only determine whether an Earth collision is possible, but also determine how close to Earth the encounter will be. Furthermore, understanding the position of an Earth encountering object on the b-plane (i.e. the uncertainty ellipsoid projected on the b-plane) is prerequisite to the keyhole analysis. The minimum distance of the unperturbed trajectory at the closest approach point on the b-plane is called the impact parameter $\mathbf{b}$ denoted by a red line segment in Figure 3. The impact parameter itself does not reveal whether the perturbed trajectory will intersect the Earth figure projected on the b-plane; however, it can be available by scaling Earth's radius $\mathrm{R}_{\oplus}$ according to Equation (2)

$$
b_{\oplus}=\mathrm{r}_{\oplus} \sqrt{1+\mathrm{v}_{\mathrm{e}}^{2} / \mathrm{v}_{\infty}^{2}}
$$

where $v_{\mathrm{e}}$ is Earth's escape velocity and $\mathrm{v}_{\infty}$ is the hyperbolic excess velocity given as follows.

$$
\begin{gathered}
\mathrm{v}_{\mathrm{e}}^{2}=2 \mathrm{GM}_{\oplus} / \mathrm{R}_{\oplus} \\
\mathrm{v}_{\infty}^{2}=\mu_{\odot}\left(3-1 / a-2 \sqrt{a\left(1-e^{2}\right)} \cos i\right)
\end{gathered}
$$

A given trajectory intersects the Earth figure on the b-plane if $\|\mathbf{b}\|$ is smaller than the scaled Earth-radius $b_{\oplus}$, and not otherwise. On the b-plane the $\xi$ coordinate is the minimum distance that can be obtained by varying the timing of the encounter. This distance, known as the minimum orbital intersection distance (MOID), is equivalent to the minimum separation between the osculating ellipses, regardless of the location of the objects on their orbits. Throughout this work, the MOID is basically set to zero and thus the incoming asteroid passes through the origin of the b-plane reference frame (i.e. the centre of Earth). 


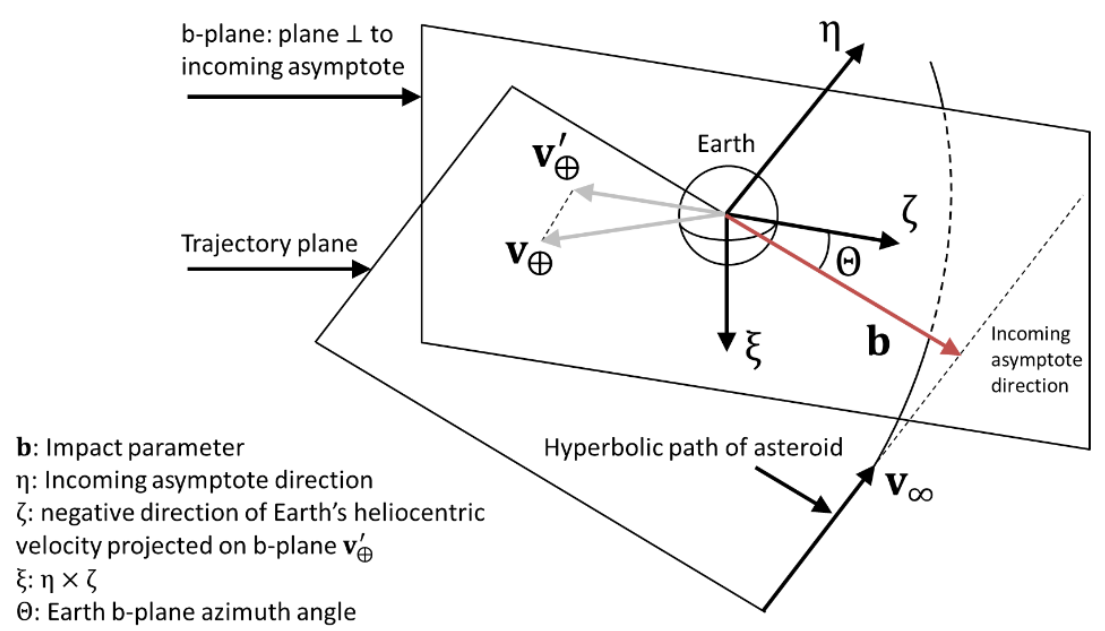

Figure 3 B-plane reference frame $(\xi, \eta, \zeta)$ on the Earth b-plane. The red arrow represents the impact parameter $b$ which is to be manipulated through an asteroid deflection mission for planetary defense purposes.

A hazardous NEA mitigation campaign therefore comes down to nudging the NEA and making $b$ at least greater than $b_{\oplus}$ on the b-plane of the impact epoch. Achieved deflections on the b-plane by the instantaneous and slow-push deflection techniques are computed by means of the proximal motion equations and Gauss' variational equations [17]. The proximal motion equations describe the variation of the position vector of the asteroid after a deflection attempt with respect to its unperturbed position, assuming the perturbed orbit is proximal to the unperturbed one. The variation vector is given as displacements in the radial, transversal, and perpendicular to the orbit plane directions, respectively. The variations of the orbital parameters of the asteroid are then computed through Gauss' variational equations. For slow-push deflection techniques, the Gauss' variational equations are numerically integrated over the deflection mission duration.

Two instantaneous deflection techniques (KI and NI) and two slow-push deflection techniques (SC and GT) are considered here. The mathematical models of these techniques are basically based on the models used in the work of Sugimoto et al. [5], which are originally developed in the work of Sanchez et al. in 2009 [1]. The modes of operation for all the mitigation systems considered in this paper are summarised in Table 2. Among them, only the KI mission intercepts the target NEA at high speed.

Table 2 Modes of operation for KI, NI, SC, and GT mitigation systems.

\begin{tabular}{|c|c|c|c|}
\hline Mitigation system & $\begin{array}{l}\text { Type of NEA } \\
\text { approach }\end{array}$ & Type of deflection action & Duration of deflection action \\
\hline KI & $\begin{array}{l}\text { high-velocity } \\
\text { interception }\end{array}$ & high-velocity impact by spacecraft & instantaneous \\
\hline NI & $\begin{array}{l}\text { orbital } \\
\text { rendezvous }\end{array}$ & $\begin{array}{l}\text { deflection by means of standoff } \\
\text { nuclear explosion of spacecraft }\end{array}$ & instantaneous \\
\hline $\mathrm{SC}$ & $\begin{array}{l}\text { orbital } \\
\text { rendezvous }\end{array}$ & $\begin{array}{l}\text { slow-push deflection through } \\
\text { sublimating asteroid material by } \\
\text { spacecraft }\end{array}$ & days to month(s) \\
\hline GT & $\begin{array}{l}\text { orbital } \\
\text { rendezvous }\end{array}$ & $\begin{array}{l}\text { slow-push deflection through } \\
\text { gravitational tractoring by spacecraft }\end{array}$ & years to decade(s) \\
\hline
\end{tabular}

In the next subsections, six virtual hazardous NEAs to be mitigated and their associated hazard scenarios are presented and the objective of NEA deflection on the b-plane is summarised. Finally, the derivation method and algorithm to represent the uncertainty of NEA deflection missions by Evidence Theory are introduced. 


\subsection{Virtual hazardous NEAs}

Virtual Earth-threatening impactors are imaginary hazardous asteroids on collision courses with Earth that will be mitigated by means of asteroid deflection in this study. Six virtual impactors $\left(\mathrm{VI}_{1}-\mathrm{VI}_{6}\right)$ were generated to represent a realistic population of impactors by taking into account the relative impact frequency of each possible trajectory [18]. The impact frequency of each trajectory was estimated by means of Öpik's collision theory [19] and Bottke's near-Earth objects orbital model [20]. The orbits of the virtual Earth impactors are shown in Figure 4. The orbit type of $\mathrm{VI}_{1}, \mathrm{VI}_{2}$, and $\mathrm{VI}_{3}$ is Apollo whereas that of $\mathrm{VI}_{4}, \mathrm{VI}_{5}$, and $\mathrm{VI}_{6}$ is Aten. The Keplerian elements of the virtual impactors are given in Table 3 .

The equivalent diameter of the virtual impactors is set to 140 metres unless otherwise stated although Earth impacts by NEAs of $>50$ metres in diameter are more frequent, and therefore more hazardous to the Earth in that sense. It is assumed, in this paper, that NEAs with 140 metres in diameter would represent the worst possible size among the subkilometre-sized NEA population, considering the insufficient NEA discovery completion rate of this size range (50-140 metres) in the foreseeable future. These relatively small NEAs can cause serious local-scale devastation on Earth far greater than the Tunguska event while they are not sufficiently discovered through the previous near-Earth object (NEO) surveys: the George E. Brown, Jr. Near-Earth Object Survey section of the 2005 NASA Authorization Mission, etc. The B612 Foundation's Sentinel mission may detect 90\% of NEOs of $>140$ metres in diameter and a fraction of those < 140 metres by 2020 [21], however the detection of $<140$-metre sized NEOs will not likely reach a 90\% discovery rate, at least in the next decade to come.

NEAs are modelled as a sphere throughout this study although it is known that asteroid shapes in nature are inhomogeneous and they are significant to asteroid deflection action of any kind. The problem of asteroid inhomogeneity is often associated with the asteroid rotation because, if the target asteroid misshapen, some asteroid characteristics (e.g. standoff distance, surface material and geometry, etc.) important to the deflection techniques change as the asteroid rotates. For example, the KI system has to carefully make its terminal approach to the asteroid, considering its rotation and shape in order to make an impact on the desired point. The NI system has to carefully choose the standoff distance and the timing of nuclear detonation according to the shape and rotation of the asteroid in order to maximise the resultant deflection or to provide the nominal deflection as planned. The SC system, on the other hand, is also subject to the asteroid shape and rotation as the concentrated solar beam has to illuminate the rotating asteroid surface and evaporate its material, resulting in inevitable fluctuations in the thrust induced by evaporation. The GT system is not entirely free from this problem as the gravity of the asteroid fluctuates as the misshapen asteroid rotates. All of these issues are unique and should be rigorously assessed for each technique as an independent study. The uncertainty in shape determination must be also taken into account in order to apply the problem to this study, which would require further preliminary study on small body shape determination and modelling techniques for instance. The asteroids are therefore treated as a spherical body for the conciseness of the paper whose focus is on mitigation campaign planning and optimisation under the uncertain information.

The Earth impact events of the virtual impactors take place on the 13th of April 2036 while each impactor is discovered and identified to be truly hazardous 10 years before the impact event, and thus a mitigation campaign will be launched and executed sometime in this 10-year warning time (i.e. between 2026/4/13 and 2036/4/13).

Although there is only $<20 \%$ chance that a $>200$-metre sized NEA would be discovered before the Earth impact given the capability of current telescopic surveillance according to the work by Morrison et al. [3], this warning time is assumed to be a reasonable period of time for 140-metre sized NEAs. 

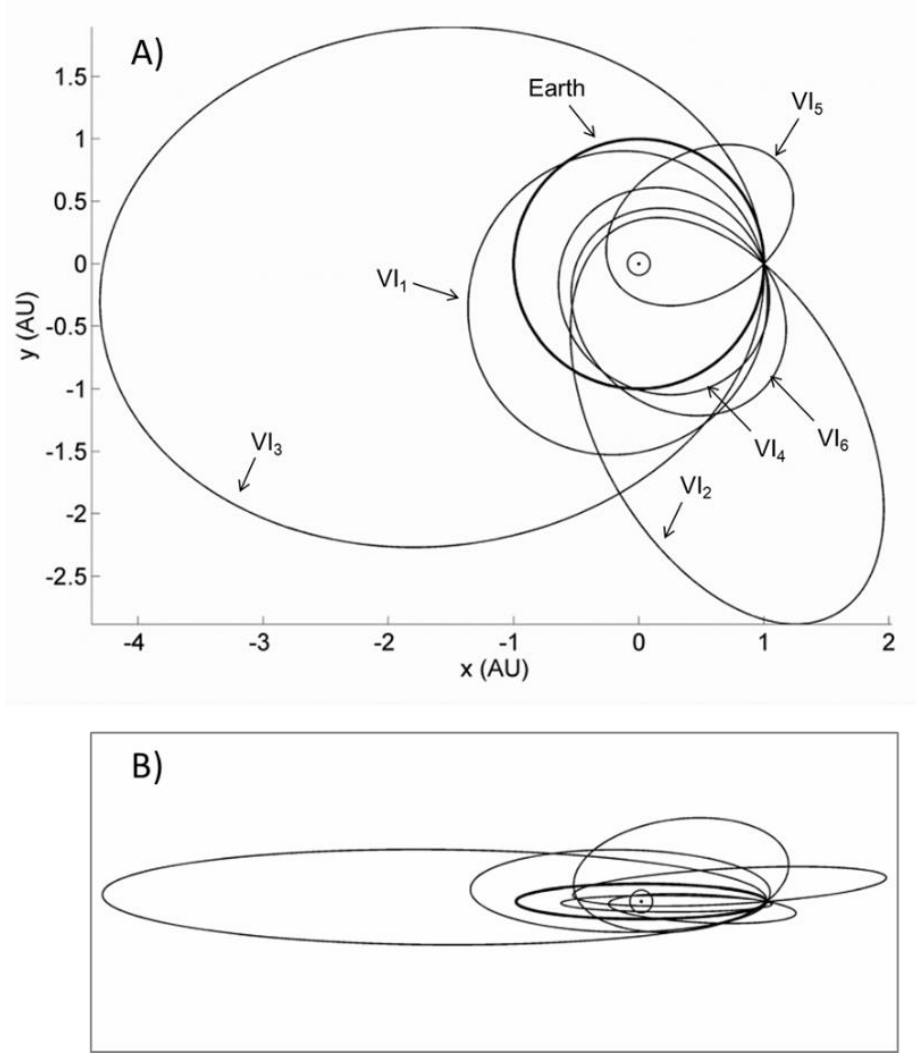

Figure 4 Orbits of virtual impactors $\mathrm{VI}_{1}-\mathrm{VI}_{6}$ and Earth. They can be categorised into two orbital groups: Apollo $\left(\mathrm{VI}_{1}, \mathrm{VI}_{2}\right.$, and $\left.\mathrm{VI}_{3}\right)$ and Aten $\left(\mathrm{VI}_{4}, \mathrm{VI}_{5}\right.$, and $\left.\mathrm{VI}_{6}\right)$ groups. A) Ecliptic plane view. B) Sideway view.

Table 3 Keplerian elements of the virtual impactors. $\theta_{\text {MOID }}$ is the true anomaly at the MOID point.

\begin{tabular}{ccccccc}
\hline Virtual impactor & $a(\mathrm{AU})$ & $e$ & $i(\mathrm{deg})$ & $\Omega(\mathrm{deg})$ & $\omega(\mathrm{deg})$ & $\theta_{\text {MOID }}(\mathrm{deg})$ \\
\hline $\mathrm{VI}_{1}$ & 1.24 & 0.289 & 7.5 & 180 & 242 & 298 \\
$\mathrm{VI}_{2}$ & 1.78 & 0.813 & 2.5 & 0 & 119 & 241 \\
$\mathrm{VI}_{3}$ & 2.66 & 0.625 & 2.5 & 180 & 186 & 354 \\
$\mathrm{VI}_{4}$ & 0.81 & 0.731 & 2.5 & 180 & 32.4 & 148 \\
$\mathrm{VI}_{5}$ & 0.87 & 0.345 & 12.5 & 0 & 132 & 228 \\
$\mathrm{VI}_{6}$ & 0.95 & 0.550 & 22.5 & 180 & 307 & 233 \\
\hline
\end{tabular}

Impact velocities with respect to Earth, and resultant energy delivered to Earth by impact of $\mathrm{VI}_{1}-\mathrm{VI}_{6}$ are summarised in Table 4. The mass of each impactor refers to the nominal value given in Table 7 . The energy delivered to Earth is therefore subject to the taxonomic class of the virtual impactors, hence three different values of resultant impact energy for each virtual impactor can be seen here.

Table 4 Impact velocities with respect to Earth, and resultant delivered energy to Earth by $\mathrm{VI}_{1}-\mathrm{VI}_{6}$.

\begin{tabular}{ccc}
\hline & Impact velocity (km/s) & $\begin{array}{c}\text { Energy delivered by impact (megatons of TNT) } \\
\text { S-type / C-type / M-type }\end{array}$ \\
\hline $\mathrm{VI}_{1}$ & 8.40 & $26.75 / 17.30 / 78.65$ \\
$\mathrm{VI}_{2}$ & 28.03 & $297.67 / 192.50 / 875.18$ \\
$\mathrm{VI}_{3}$ & 8.42 & $26.92 / 17.41 / 79.15$ \\
$\mathrm{VI}_{4}$ & 21.16 & $170.00 / 109.68 / 498.63$ \\
$\mathrm{VI}_{5}$ & 11.22 & $47.67 / 30.83 / 140.14$ \\
$\mathrm{VI}_{6}$ & 19.85 & $149.30 / 96.55 / 438.96$ \\
\hline
\end{tabular}




\subsection{Objective of NEA deflection on b-plane}

There are two kinds of deflection distances to be considered - the minimum required deflection distance and the safe deflection distance. The minimum required deflection distance is simply equivalent to $b_{\oplus}$ whereas the safe deflection distance is set to $b_{\text {safe }}=2.5$ Earth-radii throughout this work. The latter is thought to be a desired deflection distance to safely avoid an asteroid impact with Earth by the Committee to Review Near-Earth Object Surveys and Hazard Mitigation Strategies; National Research Council [2].

The objective of hazardous NEA deflection mission is therefore to make the impact parameter $b$ on the 2036 b-plane at least greater than $b_{\oplus}$, or $b_{\text {safe }}$ if possible. Table 5 shows $b_{\oplus}, b_{\text {safe, }}$, required impulsive velocity changes $\Delta v_{\oplus}$ to achieve $b_{\oplus}$, and $\Delta v_{\text {safe }}$ to achieve $b_{\text {safe }}$ for the mitigation of $\mathrm{VI}_{1-6}$, respectively. These required impulsive velocity changes (tangential to the travelling direction) are computed for a kinetic impact at the optimal interception epochs (i.e. perihelia of $\mathrm{VI}_{1-6}$ ) when a required velocity change is minimal while satisfying $\Delta b=b_{\oplus}$, given the 10-year warning time. Figure 5 shows the variation of deflection distance of $\mathrm{VI}_{1}$ on the $2036 \mathrm{~b}$-plane, given $\Delta v=0.79 \mathrm{~cm} / \mathrm{s}$ at any time between 2026 and 2036 . The velocity change should be applied on 9 October 2026 such that the b-plane deflection of one $b_{\oplus}$ can be achieved.

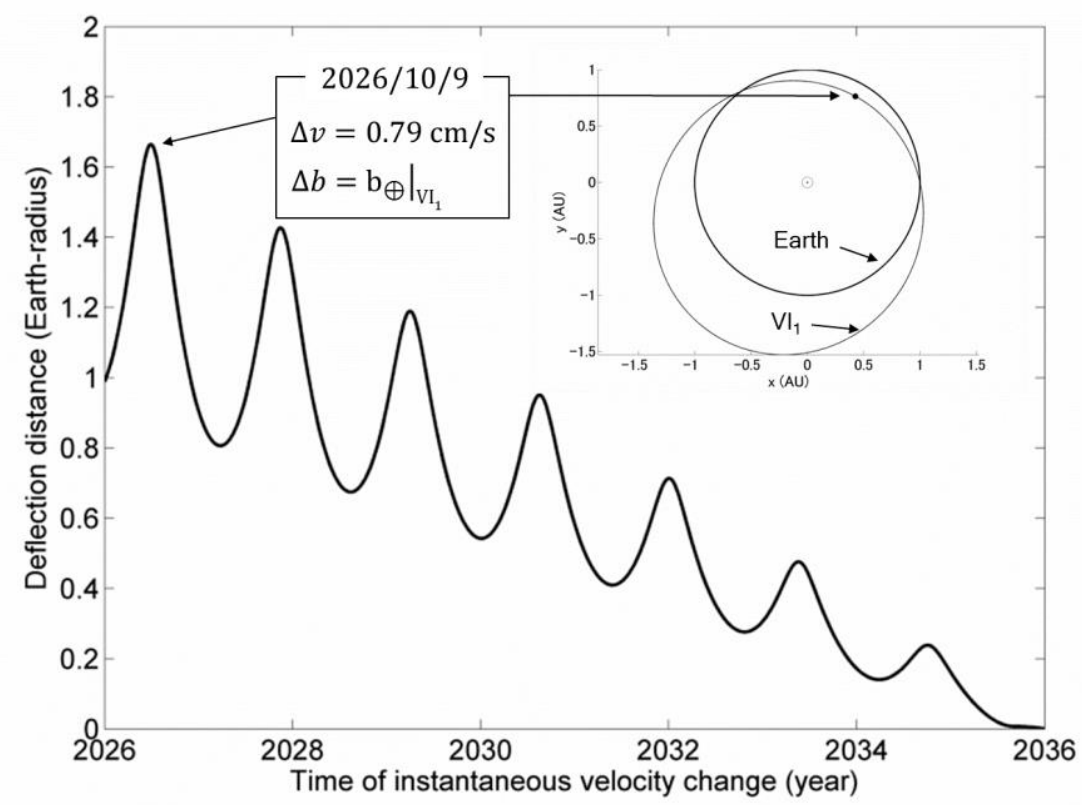

Figure 5 Variation of deflection distance of $\mathrm{VI}_{1}$ on the $2036 \mathrm{~b}$-plane, given $\Delta v=0.79 \mathrm{~cm} / \mathrm{s}$.

Table 5 Minimum deflection distance $b_{\oplus}$, safe deflection distance $b_{\text {safe }}$, impulsive velocity change $\Delta v_{\oplus}$, and the impulsive velocity change $\Delta v_{\text {safe }}$ that are required for the mitigation of $\mathrm{VI}_{1-6}$. The optimal interception epochs for respective virtual impactors range 2026/10/9-2027/8/14.

\begin{tabular}{cccccc}
\hline & $\begin{array}{c}b_{\oplus} \\
\text { (Earth-radius) }\end{array}$ & $\begin{array}{c}\Delta v_{\oplus} \\
(\mathrm{cm} / \mathrm{s})\end{array}$ & $\begin{array}{c}b_{\text {safe }} \\
\text { (Earth-radius) }\end{array}$ & $\begin{array}{c}\Delta v_{\text {safe }} \\
(\mathrm{cm} / \mathrm{s})\end{array}$ & $\begin{array}{c}\text { Interception } \\
\text { Epoch }\end{array}$ \\
\hline $\mathrm{VI}_{1}$ & 1.66 & 0.79 & 4.16 & 1.98 & $2026 / 10 / 9$ \\
$\mathrm{VI}_{2}$ & 1.08 & 0.19 & 2.70 & 0.48 & $2026 / 12 / 10$ \\
$\mathrm{VI}_{3}$ & 1.66 & 1.38 & 4.16 & 3.44 & $2027 / 8 / 14$ \\
$\mathrm{VI}_{4}$ & 1.13 & 0.43 & 2.83 & 1.07 & $2026 / 8 / 31$ \\
$\mathrm{VI}_{5}$ & 1.41 & 0.79 & 3.53 & 1.98 & $2026 / 9 / 17$ \\
$\mathrm{VI}_{6}$ & 1.15 & 0.49 & 2.87 & 1.24 & $2027 / 2 / 17$ \\
\hline
\end{tabular}




\subsection{Uncertainty in NEA deflection}

In this subsection, the performance of deflection techniques subject to epistemic uncertainties in the physical properties of the hazardous NEA is evaluated, where Evidence Theory [14] is employed to quantify the uncertainty in deflection performance. $\mathrm{KI}, \mathrm{NI}, \mathrm{SC}$, and GT are applied to $\mathrm{VI}_{1-6}$ of different taxonomic classifications (S-, C-, and M-types) at different characterisation levels (ground-based, space-based, and proximity characterisation) for reference.

Evidence Theory makes use of two probability measures called Belief $(\mathrm{Bel})$ and Plausibility $(P l)$ as illustrated in Figure 6. Belief represents confidence in the truth of an event $\mathcal{A}$ (i.e. deflection) exclusive of uncertainty while Plausibility represents confidence in the truth of the same event inclusive of uncertainty.

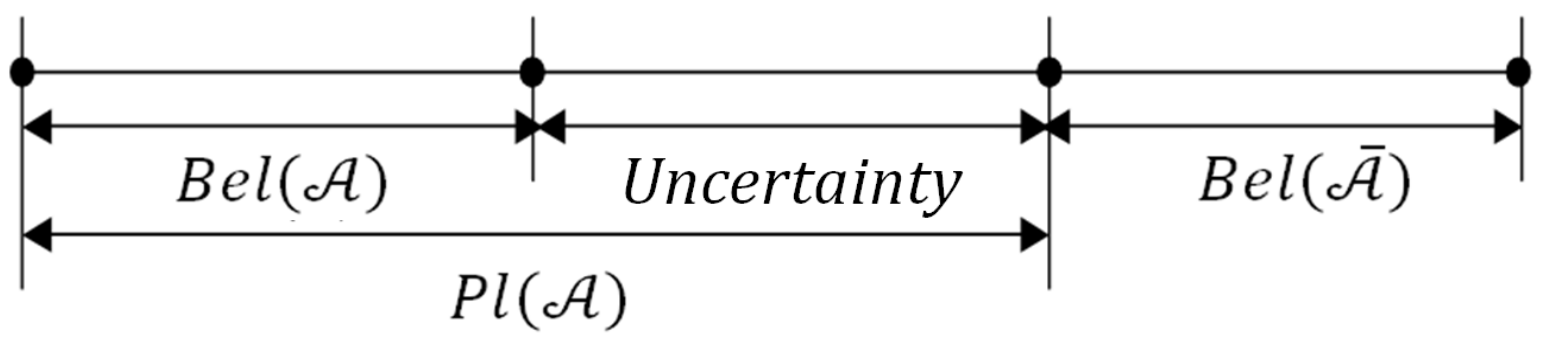

Figure 6 Belief and Plausibility [22].

Unlike probability measures of conventional probabilistic means, the sum of the Belief measure of the truth of the event $\mathcal{A}$ and that of its negation is not necessarily $(\operatorname{Bel}(\mathcal{A})+\operatorname{Bel}(\overline{\mathcal{A}}) \leq 1)$. The two probability measures are therefore subject to the uncertain NEA characteristics in different ways; however, the more rigorous the preliminary characterisation is, the less different the two measures will be. Belief informs the lower bounds of deflection outcomes whereas Plausibility informs the upper bounds of deflection outcomes. To this extent, Belief is found to be useful for more strict assessment of confidence level on deflection missions.

In order to evaluate Belief and Plausibility of a given deflection, basic probability assignment (BPA) structures for the NEA physical properties must be assembled (see Appendix A.1 for further details). A BPA $m(\mathcal{E})$ is a basic uncertainty measure which represents the degree of confidence in the truth of an event $\mathcal{E}$ (i.e. specific NEA physical property) and satisfies the following three axioms:

$$
\begin{gathered}
m(\mathcal{E}) \geq 0 \text { for any } \mathcal{E} \in 2^{u} \\
m(\emptyset)=0 \\
\sum m(\mathcal{E})=1 \text { for all } \mathcal{E} \in 2^{u}
\end{gathered}
$$

where $\emptyset$ denotes an empty set. All the events $\mathcal{E}$ that are subsets of the universal set $\mathcal{U}(\mathcal{E} \subseteq \mathcal{U})$ and those which have $m(\mathcal{E})>0$ are known as the focal elements.

Epistemic uncertainties and parametric uncertainties (i.e. measurement errors) in a given parameter are described as a set of intervals with associated BPAs. Figure 7 is a schematic representation of such a BPA 
structure for the uncertain parameter $p_{1}$. In Table 6 , the intervals for $p_{1}$ are non-nested with each other, however in general, they can be nested. These interval values are usually obtained from observations and experiments as well as from expert opinions and hypotheses (see Appendix A.1 for further details).

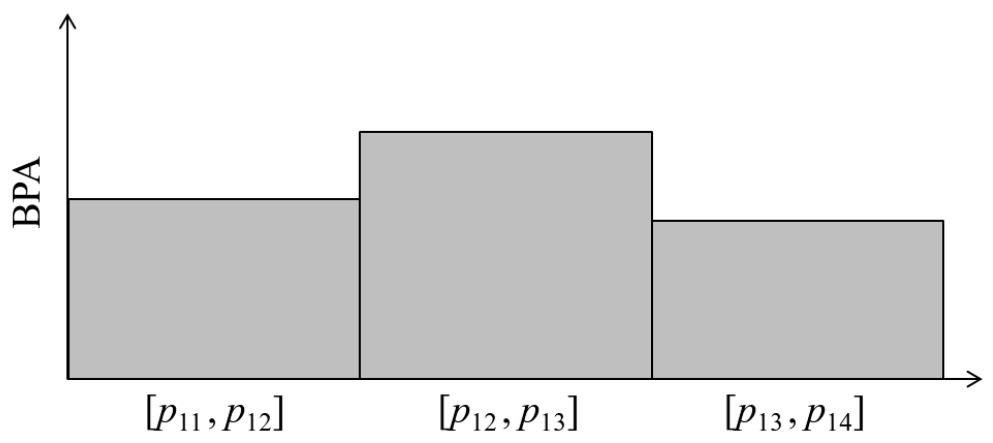

Figure 7 BPA structure for the uncertain parameter $p_{1}$.

Table 6 Intervals with associated BPAs for the uncertain parameter $p_{1}$.

\begin{tabular}{ccc}
\hline Uncertain parameter & Interval & BPA \\
\hline$p_{1}$ & {$\left[p_{11}, p_{12}\right]$} & $m_{11}$ \\
& {$\left[p_{12}, p_{13}\right]$} & $m_{12}$ \\
& {$\left[p_{13}, p_{14}\right]$} & $m_{13}$ \\
\hline
\end{tabular}

Evidence-based information from different sources that supports the truth of an event $\mathcal{A}$ can be aggregated using existing rules of combination. In this study, the Dempster rule of combination [22] given in Equation (8) is used, where $m_{1}(\mathcal{B})$ and $m_{2}(\mathcal{C})$ are the degrees of confidence in the truth of an event $\mathcal{B}$ and that in the truth of an event $\mathcal{C}$, respectively.

$$
m(\mathcal{A})=\frac{\sum_{\mathcal{B} \cap \mathcal{C}=\mathcal{A}} m_{1}(\mathcal{B}) m_{2}(\mathcal{C})}{1-\sum_{\mathcal{B} \cap \mathcal{C} \neq \emptyset} m_{1}(\mathcal{B}) m_{2}(\mathcal{C})}, \mathcal{A} \neq \emptyset
$$

Belief is then computed by summing the BPAs of events $\mathcal{B}$ that totally support the truth of the event $\mathcal{A}$ while Plausibility is computed by summing the BPAs of events $\mathcal{B}$ which totally or partly support the truth of the event $\mathcal{A}$ as shown Equations (9) and (10), respectively. In other words, Belief and Plausibility inform the lower and upper bounds of the event $\mathcal{A}$ respectively. They pertain to each other by Equation (11).

$$
\begin{gathered}
\operatorname{Bel}(\mathcal{A})=\sum_{\mathcal{B} \mid \mathcal{B} \subseteq \mathcal{A}} m(\mathcal{B}) \\
\operatorname{Pl}(\mathcal{A})=\sum_{\mathcal{B} \mid \mathcal{B} \cap \mathcal{A} \neq \emptyset} m(\mathcal{B}) \\
\operatorname{Pl}(\mathcal{A})+\operatorname{Bel}(\overline{\mathcal{A}})=1
\end{gathered}
$$

In this work, the following steps are performed to evaluate Belief and Plausibility of asteroid deflection:

a) Collect all the necessary information on the asteroid characteristics from different literature (see Appendix A.1 for further details on information gathering). 
b) Determine the nominal physical properties of the NEA from the information collected in step a) as shown in Table 7, and design a mitigation system based on this baseline for the nominal deflection distance $b_{\text {nom }}$ within $b_{\oplus}<b_{\text {nom }}<b_{\text {safe }}$. The nominal deflection $b_{\text {nom }}$ is a b-plane deflection that will be achieved through the given deflection mission to the NEA actually bearing the nominal physical properties.

c) Build BPA structures of the NEA characteristics by defining each uncertainty parameter as a set of intervals with prescribed BPAs and, if necessary, integrate BPA structures of the same parameter from different sources by means of the Dempster rule of combination. Those of micro density $\rho_{\text {micro }}$, micro porosity $P_{\text {micro }}$, bulk porosity $P_{\text {bulk }}$, and albedo $p_{v}$ are shown in Table 12 in Appendix A.1.

d) Calculate a set $C_{i}$ of uncertain intervals of the NEA mass $M_{a}$ and momentum enhancement factor $\beta$ with associated degree of confidence $m_{c}\left(C_{i}\right)$ which is simply the product of BPAs of related physical properties that support the truth of the set $C_{i}$.

e) Given the mitigation system designed in step b) and the information on the set $C_{i}$ of $M_{a}$ and $\beta$ with $m_{c}\left(C_{i}\right)$ from step d), compute the possible deflection $b$ for the case of set $C_{i}$. Run over step d) and step e) for all the possible sets of $M_{a}$ and $\beta$. As an example, Table 8 shows 16 sets of $M_{a}, \beta, \Delta v$, and $b$.

f) Computed Belief $\operatorname{Bel}(b)$ and Plausibility $P l(b)$ of an arbitrary deflection $b$ by Equations (12) and (13).

$$
\begin{gathered}
\operatorname{Bel}(b)=\sum_{C_{i} \mid C_{i} \subseteq b} m_{c}\left(C_{i}\right) \\
P l(b)=\sum_{C_{i} \mid C_{i} \cap b \neq \emptyset} m_{c}\left(C_{i}\right)
\end{gathered}
$$

In step b), the mitigation system is designed based on the nominal physical properties instead of considering a system margin approach or the worst-possible combination of NEA characteristics (e.g. largest micro-density, smallest bulk-porosity, highest albedo, etc. of each taxonomic class). The reason for this is because changing the design parameters does not contributed to the inherent uncertainty in deflection outcome subject to the epistemic uncertainties in the NEA characteristics. Of course, it is obvious that, if the mitigation system is designed in consideration of the system margin, the system can provided a nominal deflection with the asteroid, given the worst-possible combination of NEA characteristics. The resultant deflection is however still ambiguous due to the epistemic uncertainties originating from the incomplete preliminary NEA characterisation.

Table 8 shows an example of BPA structures for the NEA mass $M_{a}$, momentum enhancement factor $\beta$, deflection $b$ on the 2036 b-plane, and impulsive velocity change $\Delta v$ given by KI. In this case, the nominal deflection $b_{\text {nom }}$ is set to 2.5 Earth-radii and the target NEA is $\mathrm{VI}_{1}$ of S-type characterised at the ground-based level. $M_{a}$ can be calculated simply by Equation (1) whereas $\beta$ is obtained by a solution of Equation (14) originating from the work by Holsapple et al. [23].

$$
\beta=1+(0.083) U^{0.2} Y^{-0.1} \rho_{\text {bulk }}^{-0.1} \delta^{0.2}
$$

where $U$ is impactor velocity, $Y$ is the material strength of the target asteroid, and $\delta$ is the impactor density. The material strength of S-type is assumed to be $10 \mathrm{kPa}$ and the impactor density is set to $3.0 \mathrm{~g} / \mathrm{cm}^{3}$. The bulk density is given by $\rho_{\text {bulk }}=\rho_{\text {micro }}\left(1-P_{\text {bulk }}\right)$. The impactor velocity for the case of KI is one of the design variables, hence is simply subject to the mitigation system design. 
Table 7 Nominal physical properties of three asteroid classes; micro density $\rho_{\text {micro }}$, micro porosity $P_{\text {micro }}$, bulk density $\rho_{\text {bulk }}$, bulk porosity $P_{\text {bulk }}$, geometric albedo $p_{v}$, and NEA mass $M_{a}$. A) Ground-based characterisation scenario. B) Space-based characterisation scenario. C) Proximity characterisation scenario.

\begin{tabular}{lcccccc}
\hline A) & $\rho_{\text {micro }}\left(\mathrm{g} / \mathrm{cm}^{3}\right)$ & $P_{\text {micro }}(\%)$ & $\rho_{\text {bulk }}\left(\mathrm{g} / \mathrm{cm}^{3}\right)$ & $P_{\text {bulk }}(\%)$ & $p_{v}$ & $M_{a}(\mathrm{~kg})$ \\
\hline S-type & 3.45 & 10.66 & 2.20 & 36.17 & 0.19 & $3.17 \times 10^{9}$ \\
C-type & 2.71 & 23.00 & 2.21 & 47.36 & 0.05 & $2.05 \times 10^{9}$ \\
M-type & 7.87 & 0.60 & 2.27 & 17.55 & 0.12 & $9.32 \times 10^{9}$ \\
\hline B) & $\rho_{\text {micro }}\left(\mathrm{g} / \mathrm{cm}^{3}\right)$ & $P_{\text {micro }}(\%)$ & $\rho_{\text {bulk }}\left(\mathrm{g} / \mathrm{cm}^{3}\right)$ & $P_{\text {bulk }}(\%)$ & $p_{v}$ & $M_{a}(\mathrm{~kg})$ \\
\hline S-type & 3.45 & 10.66 & 1.42 & 36.12 & 0.18 & $3.17 \times 10^{9}$ \\
C-type & 2.71 & 23.00 & 1.43 & 47.34 & 0.05 & $2.05 \times 10^{9}$ \\
M-type & 7.87 & 0.60 & 1.47 & 17.45 & 0.12 & $9.33 \times 10^{9}$ \\
\hline C) & $\rho_{\text {micro }}\left(\mathrm{g} / \mathrm{cm}^{3}\right)$ & $P_{\text {micro }}(\%)$ & $\rho_{\text {bulk }}\left(\mathrm{g} / \mathrm{cm}^{3}\right)$ & $P_{\text {bulk }}(\%)$ & $p_{v}$ & $M_{a}(\mathrm{~kg})$ \\
\hline S-type & 3.45 & 10.89 & 6.49 & 34.35 & 0.19 & $3.26 \times 10^{9}$ \\
C-type & 2.70 & 23.00 & 6.49 & 45.71 & 0.05 & $2.11 \times 10^{9}$ \\
M-type & 7.88 & 0.60 & 6.85 & 13.14 & 0.12 & $9.84 \times 10^{9}$ \\
\hline
\end{tabular}

Table 8 Example of BPA structures for NEA mass $M_{a}$, momentum enhancement factor $\beta$, deflection $b$ on the 2036 b-plane, and the impulsive velocity change $\Delta v$ by KI with impact velocity of $16.4 \mathrm{~km} / \mathrm{s}$. $b_{\text {nom }}$ is set to 2.5 Earth-radii and the target $\mathrm{NEA}$ is $\mathrm{VI}_{1}$ of S-type characterised at the ground-based level.

\begin{tabular}{cccccccccc}
\hline & \multicolumn{2}{c}{$M_{a}(\mathrm{~kg})$} & \multicolumn{2}{c}{$\beta$} & \multicolumn{2}{c}{$b$ (Earth-radius) } & \multicolumn{2}{c}{$\Delta v(\mathrm{~cm} / \mathrm{s})$} & $m_{c}\left(C_{i}\right)$ \\
& $\begin{array}{c}\text { Lower } \\
\text { bound }\end{array}$ & $\begin{array}{c}\text { Upper } \\
\text { bound }\end{array}$ & $\begin{array}{c}\text { Lower } \\
\text { bound }\end{array}$ & $\begin{array}{c}\text { Upper } \\
\text { bound }\end{array}$ & $\begin{array}{c}\text { Lower } \\
\text { bound }\end{array}$ & $\begin{array}{c}\text { Upper } \\
\text { bound }\end{array}$ & $\begin{array}{c}\text { Lower } \\
\text { bound }\end{array}$ & $\begin{array}{c}\text { Upper } \\
\text { bound }\end{array}$ \\
\hline$C_{1}$ & $2.02 \times 10^{9}$ & $2.37 \times 10^{9}$ & 1.55 & 1.54 & 3.33 & 3.93 & 2.33 & 2.75 & 0.04 \\
$C_{2}$ & $2.07 \times 10^{9}$ & $2.51 \times 10^{9}$ & 1.55 & 1.54 & 3.14 & 3.84 & 2.20 & 2.69 & 0.04 \\
$C_{3}$ & $2.19 \times 10^{9}$ & $2.66 \times 10^{9}$ & 1.55 & 1.54 & 2.96 & 3.62 & 2.07 & 2.53 & 0.05 \\
$C_{4}$ & $2.32 \times 10^{9}$ & $2.76 \times 10^{9}$ & 1.55 & 1.54 & 2.85 & 3.41 & 1.99 & 2.39 & 0.01 \\
$C_{5}$ & $2.32 \times 10^{9}$ & $2.85 \times 10^{9}$ & 1.55 & 1.53 & 2.76 & 3.41 & 1.93 & 2.39 & 0.09 \\
$C_{6}$ & $2.37 \times 10^{9}$ & $3.02 \times 10^{9}$ & 1.54 & 1.53 & 2.60 & 3.33 & 1.82 & 2.33 & 0.08 \\
$C_{7}$ & $2.51 \times 10^{9}$ & $3.19 \times 10^{9}$ & 1.54 & 1.53 & 2.45 & 3.14 & 1.72 & 2.20 & 0.09 \\
$C_{8}$ & $2.66 \times 10^{9}$ & $3.31 \times 10^{9}$ & 1.54 & 1.53 & 2.36 & 2.96 & 1.65 & 2.07 & 0.02 \\
$C_{9}$ & $2.78 \times 10^{9}$ & $3.32 \times 10^{9}$ & 1.54 & 1.53 & 2.35 & 2.82 & 1.65 & 1.98 & 0.04 \\
$C_{10}$ & $2.85 \times 10^{9}$ & $3.52 \times 10^{9}$ & 1.53 & 1.52 & 2.21 & 2.76 & 1.55 & 1.93 & 0.04 \\
$C_{11}$ & $3.02 \times 10^{9}$ & $3.72 \times 10^{9}$ & 1.53 & 1.52 & 2.09 & 2.60 & 1.46 & 1.82 & 0.05 \\
$C_{12}$ & $3.19 \times 10^{9}$ & $3.86 \times 10^{9}$ & 1.53 & 1.52 & 2.01 & 2.45 & 1.41 & 1.72 & 0.01 \\
$C_{13}$ & $3.25 \times 10^{9}$ & $3.96 \times 10^{9}$ & 1.53 & 1.52 & 1.96 & 2.41 & 1.37 & 1.68 & 0.13 \\
$C_{14}$ & $3.32 \times 10^{9}$ & $4.20 \times 10^{9}$ & 1.53 & 1.51 & 1.84 & 2.35 & 1.29 & 1.65 & 0.12 \\
$C_{15}$ & $3.52 \times 10^{9}$ & $4.44 \times 10^{9}$ & 1.52 & 1.51 & 1.74 & 2.21 & 1.22 & 1.55 & 0.14 \\
$C_{16}$ & $3.72 \times 10^{9}$ & $4.61 \times 10^{9}$ & 1.52 & 1.51 & 1.68 & 2.09 & 1.17 & 1.46 & 0.03 \\
\hline
\end{tabular}


The uncertainty in the outcomes (i.e. deflection distance $b$ ) of respective deflection techniques represented by the Belief and Plausibility measures are shown in Figure 8, Figure 9, and summarised in Table 9. Care must be taken when interpreting the meaning of these results: the mitigation systems here are designed arbitrarily such that the resultant deflection satisfies the nominal deflection distance of 2.5 Earth-radii when the asteroid actual physical properties are identical to the nominal design parameters (i.e. nominal/baseline physical properties defined previously in Table 7). These results are therefore not particularly useful for efficiency analysis but they are merely for reliability analysis. For example, the GT system used to obtain the results assumed hugely massive GT spacecraft in order to provide the nominal deflection of 2.5 Earth-radii, given the 10-year warning time. Firstly, it can be seen that Belief and Plausibility measures inform lower and upper bounds of the outcomes of deflection missions. Such straightforward information is available through a conventional system margin approach which only considers a series of possible ranges of system design parameters (e.g. $M_{a}$ and $\beta$ ) without assigning probabilities (i.e. deflection outcomes are always given as a plain interval value without carrying any information about epistemic uncertainties within the interval.). On the other hand, these probability measures associated with Evidence Theory indicate confidence in every possible outcome of deflection missions, which is directly related to the source of uncertainty and current uncertainty level. To this extent, Evidence Theory allows a more rigorous quantification of uncertainties than the aforementioned system margin approach does.

The results indicate that no matter how much literature or how many expert opinions one refers to for mitigation system design, as long as there are epistemic uncertainties and measurement errors in observational data, the uncertainty in deflection will be present. In other words, for whatever deflection $b$ with $\operatorname{Bel}(b)<1.0$, the confidence in achieving that deflection distance is to a greater or lesser extent, compromised.

Depending on the physical properties that are related to each deflection technique and also the taxonomic class of the asteroid, Belief/Plausibility of the deflection varies. Due to the substantial amount of uncertainty in albedo shown in Table 1, the uncertainty range of b-plane deflection achieved through the SC is considerably wide as seen in Figure 9-C), although belief and plausibility of the nominal deflection remain 0.4-0.6, similarly to the KI or the NI. Figure 8-A) and Figure 8-B) indicate that the KI and the NI are subject to more or less the same amount of uncertainty. This can be interpreted as both KI and NI are instantaneous deflection techniques that are dependent on the uncertainties in the mass and the momentum multiplication of the NEA but independent of the albedo value. It should be noted that, for the standoff NI deflection model considered in this paper, the momentum multiplication becomes of importance when dealing with the contribution of a fraction of high-velocity scattering debris of the NI spacecraft impinging on the asteroid surface. It is obvious that the NI is the most efficient NEA deflection technique in terms of the yield-to-weight (YTW) ratio among the four techniques but can never be the most accurate unless the target body is well-characterised through the proximity characterisation. Such a drawback of the NI may be minor and easily overcome by taking system margin into consideration and adjusting the standoff distance (i.e. trimming deflection leverage) before detonation, after pre-detonation in-situ characterisation of the target body by the NI spacecraft, itself.

Figure 9-D) shows a series of results of the GT mission, where the amount of uncertainty in deflection is clearly smallest among the four deflection techniques in any characterisation scenarios as well as for any types of asteroid. These results indicate that the performance of GT is least dependent on the epistemic uncertainties in NEA physical characteristics, particularly on the uncertainty in mass. The reason for this is because the overall deflection efficiency of the GT system is dependent on the NEA mass, canting angle of ion thrusters, standoff distance, and the mission duration. Given the GT mission duration of 10 years and considering only one specific asteroid type, the optimal standoff distance, hence the canting angle of the ion thrusters slightly changes as the 
asteroid mass increases/decreases from the nominal value within its uncertain range. The change in the canting angle, in turn, results in the change in the ion thrusters' output for hovering. For example, if the actual asteroid mass is smaller/larger than the nominal value, the standoff distance should be smaller/larger in order to provide the nominal deflection leverage $\left(\mathrm{F}=m_{\mathrm{GT}} / \mathrm{R}^{2}\right)$, which affects the canting angle, hence the required ion thrusters' output for hovering. As a consequence, the deflection efficiency does not substantially increase/degrease due to the smaller/larger asteroid mass with respect to the nominal value, but instead, remains alike (compared with the nominal deflection efficiency). It should be noted however that the GT system is the least efficient deflection technique among the deflection techniques being evaluated, due to its low YTW ratio (e.g. hugely massive, 10-20 tons of GT system mass is required to provide the NEA with the b-plane deflection of 2.5 Earth-radii, given 10 years of warning time) and long mission duration. To this extent, a GT would be suitable for a secondary deflection mission that backs up a more unpredictable but efficient instantaneous deflection attempt.

In summary, unless the preliminary characterisation is conducted at the proximity characterisation level, the outcome of a NEA deflection mission will always lack precision, whatever the design parameters (e.g. nominal NEA characteristics) and the design variables (e.g. mitigation system mass, impact velocity, collector size, etc.) are selected, hence the deflection performance will be compromised. Considering a system margin (i.e. increasing the deflection performance of a single mitigation system) in order to ensure $\operatorname{Bel}\left(b_{\text {safe }}\right)=1.0$ is valid here, however the aim of this work is not to design a single mitigation system that is capable of providing a desired deflection at its worst possible performance but to develop a mitigation campaign consisting of multi-deflection mission that retains a certain level of confidence in achieving a desired deflection even if the primary deflection mission is unsuccessful.

Table 9 Lower and upper bounds of the deflection $b$ (Earth-radius) on the 2036 b-plane for different deflection techniques, characterisation scenarios, and taxonomic classes. A) KI. B) NI. C) SC. D) GT.

\begin{tabular}{lccc}
\hline A) & S-type & C-type & M-type \\
\hline Ground-based & $1.70-3.97$ & $1.70-4.38$ & $2.05-3.33$ \\
Space-based & $1.81-3.55$ & $1.83-3.75$ & $2.11-3.11$ \\
Proximity & $2.29-2.66$ & $2.38-2.61$ & $2.45-2.57$ \\
\hline B) & S-type & C-type & M-type \\
\hline Ground-based & $1.71-3.93$ & $1.71-4.32$ & $2.01-3.33$ \\
Space-based & $1.82-3.52$ & $1.84-3.71$ & $2.11-3.12$ \\
Proximity & $2.29-2.65$ & $2.34-2.62$ & $2.46-2.57$ \\
\hline C) & S-type & C-type & M-type \\
\hline Ground-based & $1.11-5.23$ & $1.60-4.60$ & $1.46-4.54$ \\
Space-based & $1.34-4.23$ & $1.74-3.90$ & $1.61-3.99$ \\
Proximity & $2.15-2.85$ & $2.35-2.63$ & $2.35-2.74$ \\
\hline D) & S-type & C-type & M-type \\
\hline Ground-based & $2.32-2.81$ & $2.38-2.85$ & $2.25-2.86$ \\
Space-based & $2.35-2.79$ & $2.41-2.80$ & $2.29-2.77$ \\
Proximity & $2.48-2.56$ & $2.49-2.54$ & $2.48-2.54$ \\
\hline
\end{tabular}


A)
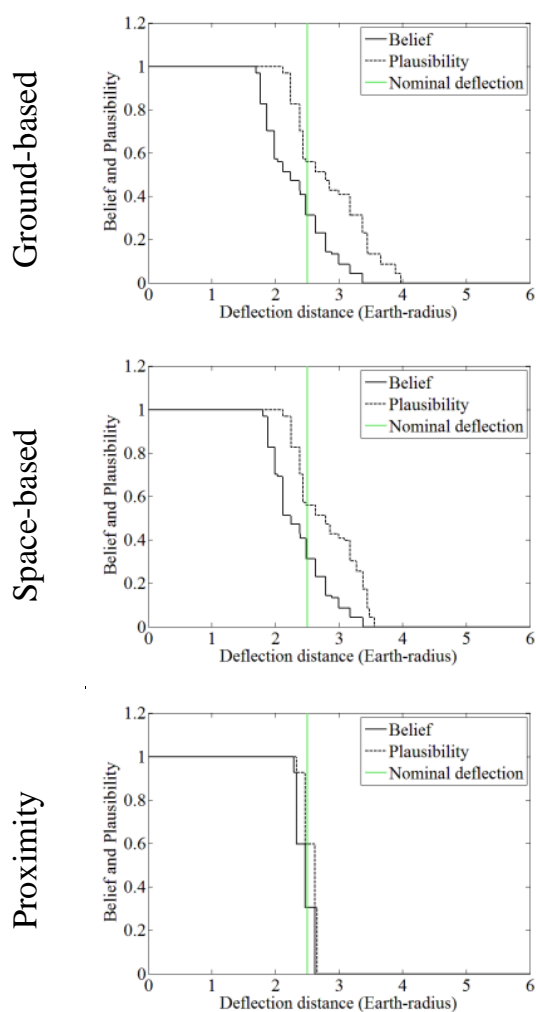

B)
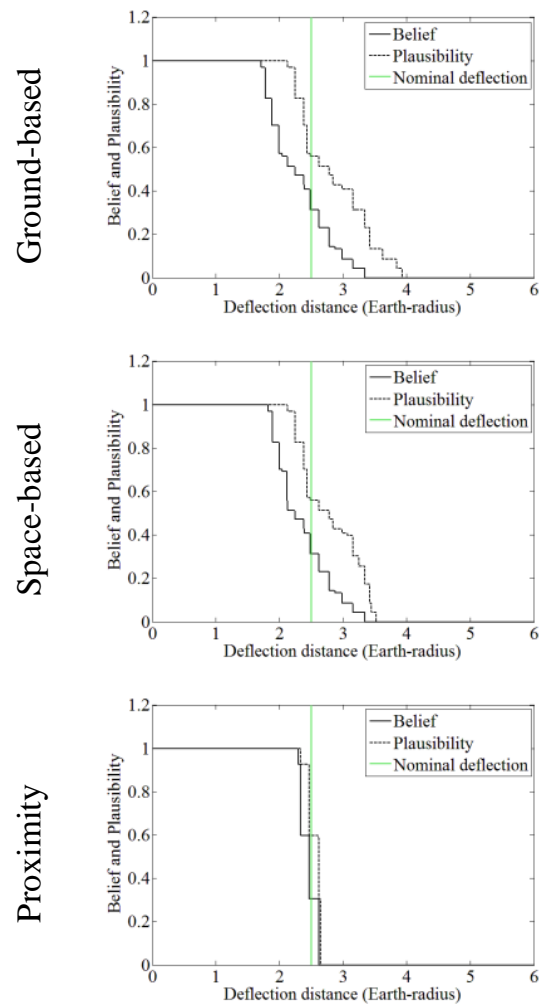

C-type
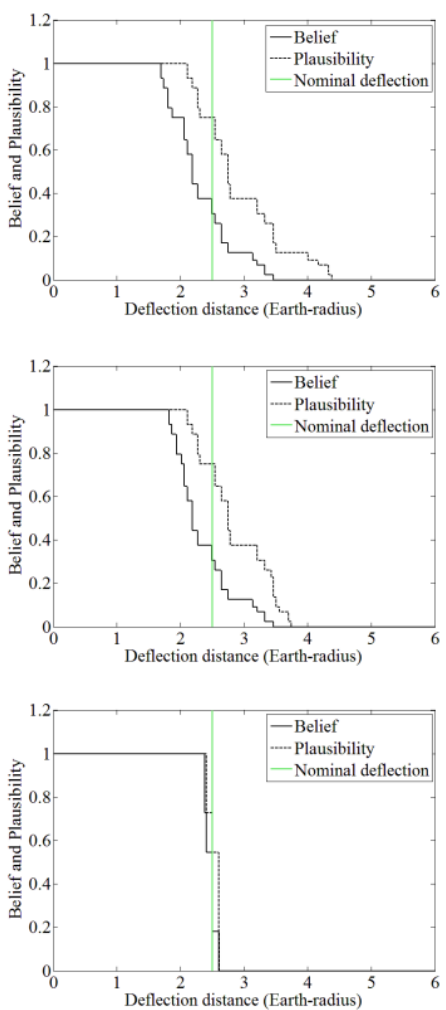

C-type
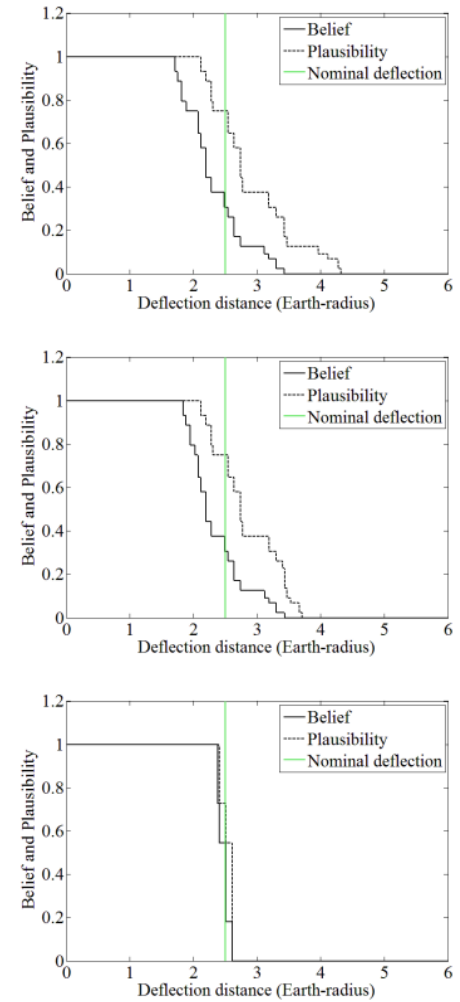

M-type
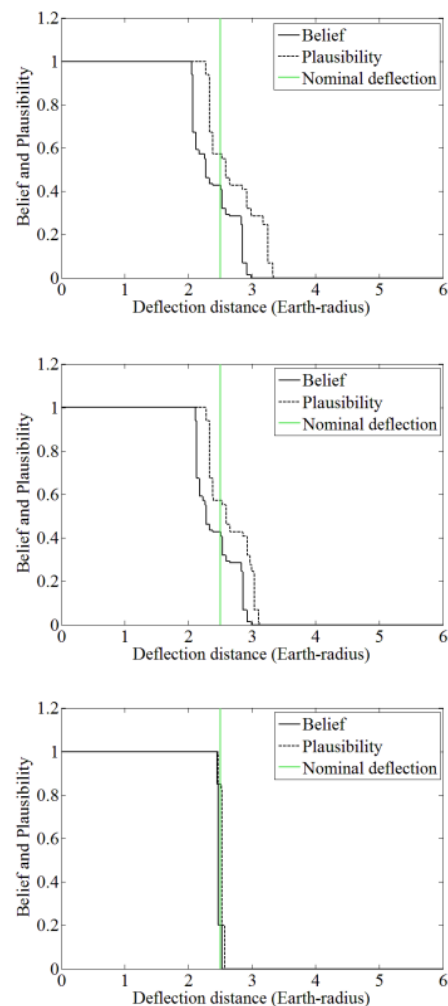

M-type
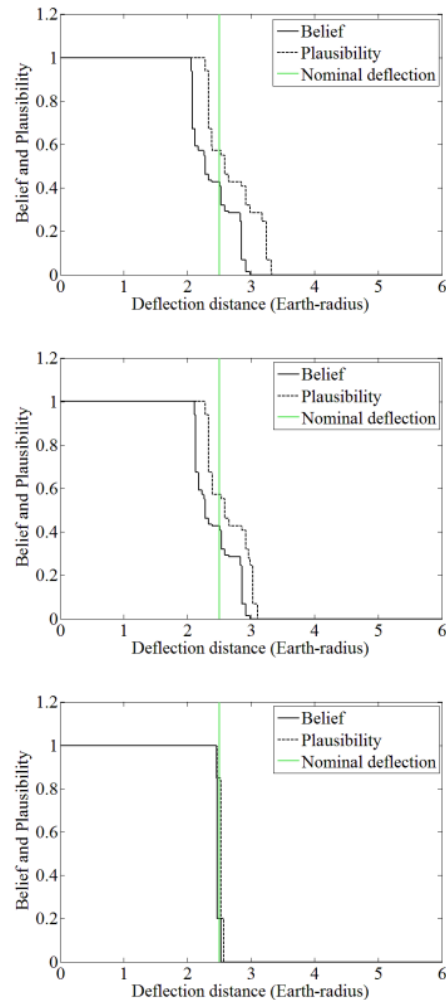

Figure 8 Belief and Plausibility of deflection on the 2036 b-plane for different characterisation scenarios and asteroid classes. Black lines represent Belief measures whereas dashed black lines represent Plausibility measures. Green lines represent the nominal deflection distance $b_{\text {nom }}$. A) KI. B) NI. 
C)
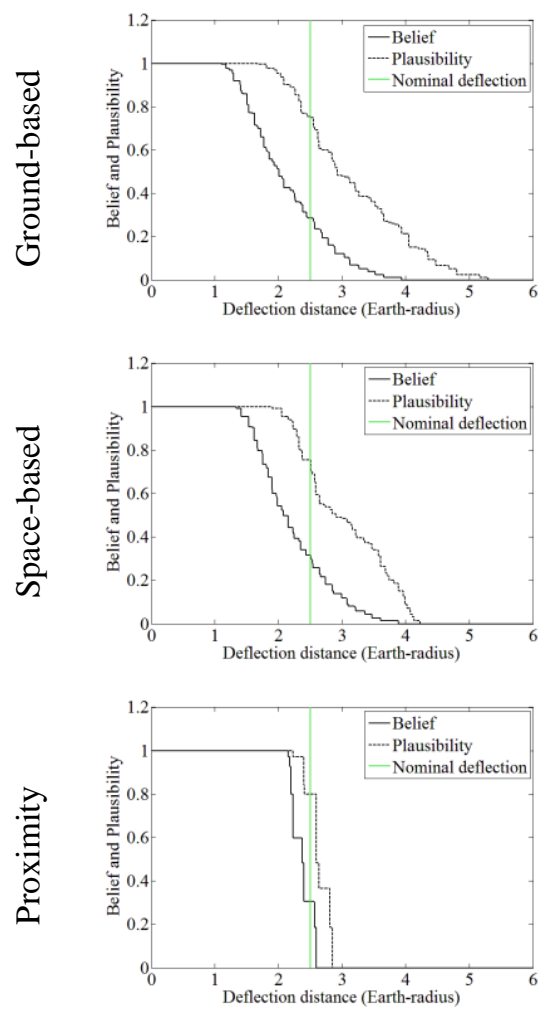

D)
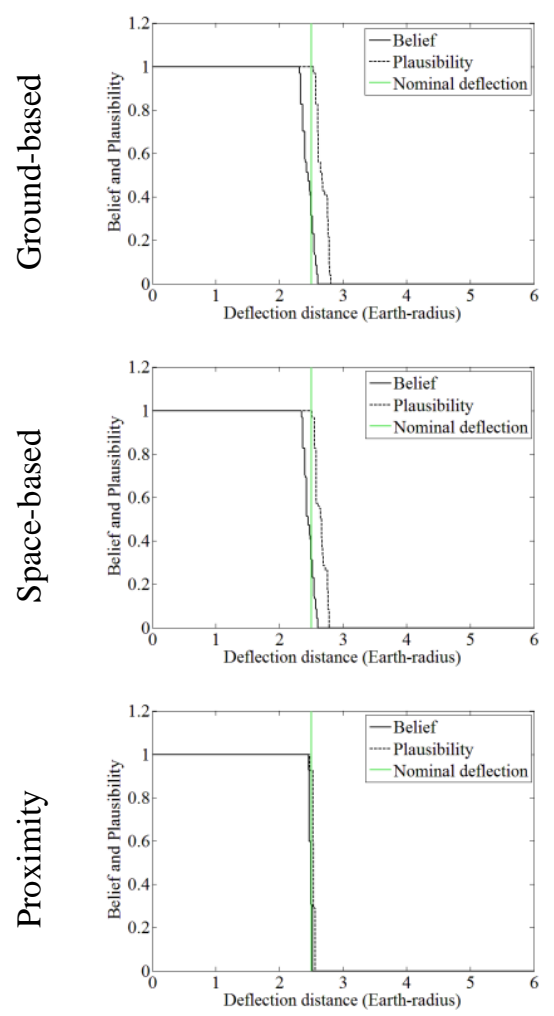

C-type
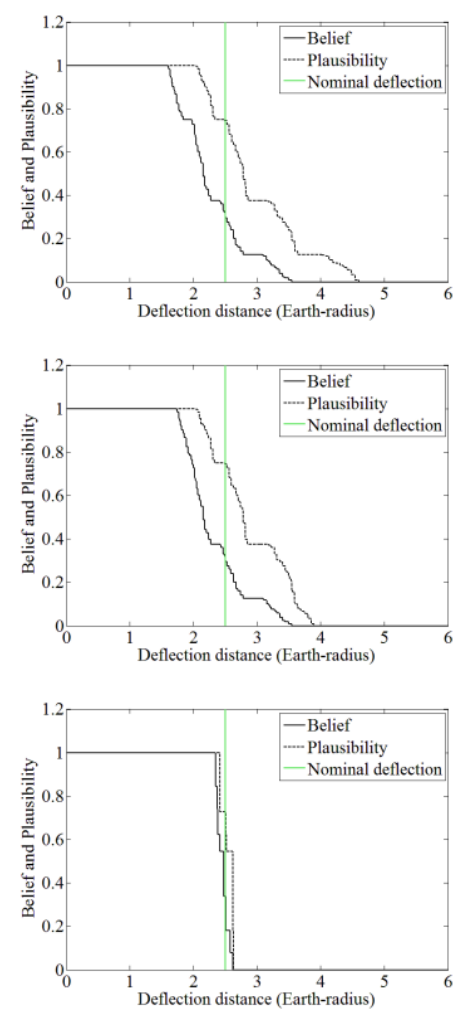

C-type
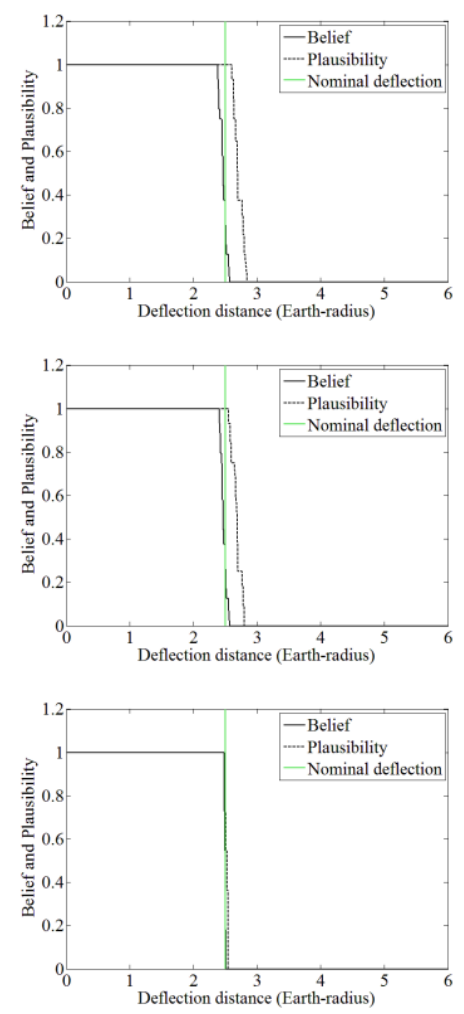

M-type
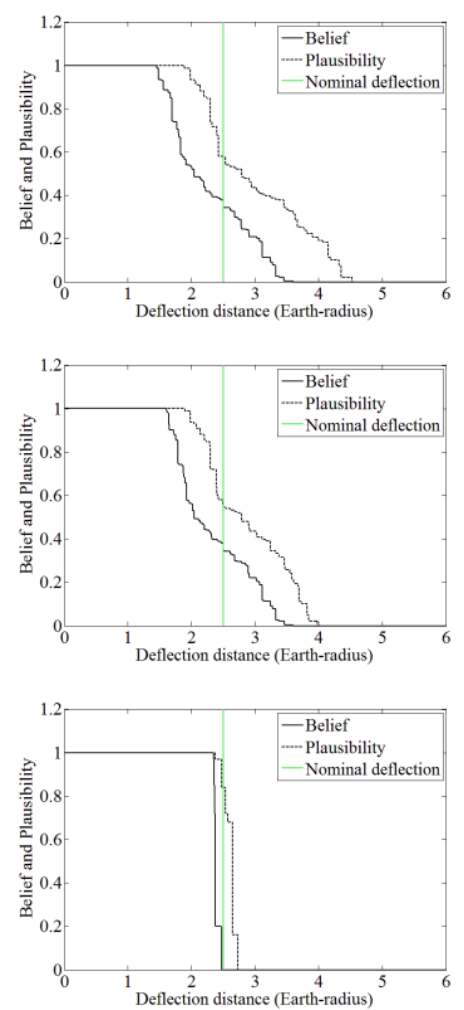

M-type
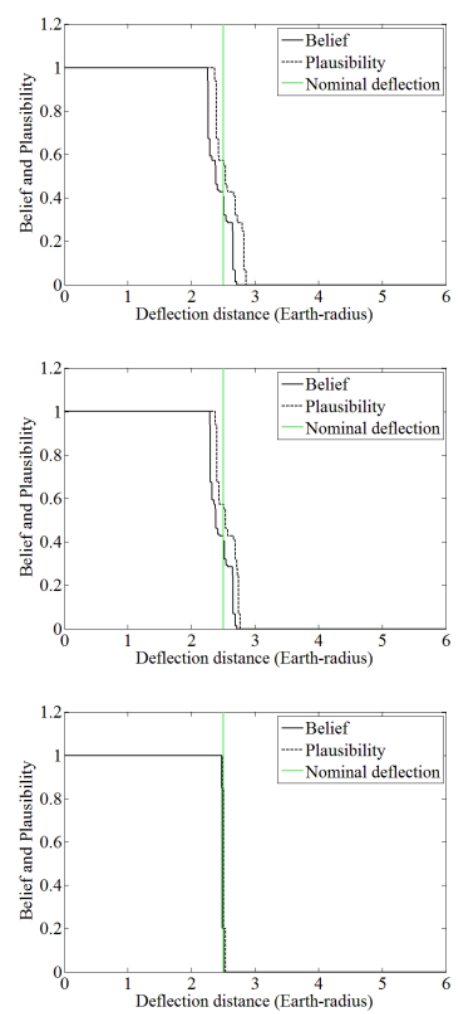

Figure 9 Belief and Plausibility of deflection on the 2036 b-plane for different characterisation scenarios and asteroid classes. Black lines represent Belief measures whereas dashed black lines represent Plausibility measures. Green lines represent the nominal deflection distance $b_{\text {nom }}$. C) SC. D) GT. 


\section{Mitigation campaign planning}

Mitigation campaigns should be accurately planned in order to provide a successful deflection even if the preliminary NEA characterisation is incomplete. Sending multiple spacecraft of one specific type of deflection technique (e.g. the multiple solar mirror concept of Maddock et al. [24] and the multiple GT concept of Foster et $a l$. [25]) can increase the deflection efficiency as well as the redundancy of given deflection mission. However, such mitigation campaigns are inevitably subject to the uncertain performance of a specific deflection technique due to not only the epistemic uncertainties in the NEA characteristics but aleatory/practical uncertainties in the technique (e.g. the precision of a KI, the time-variable sublimation efficiency of a SC, etc.).

Sending multiple asteroid deflection missions of different techniques is one of the answers to the design of a credible mitigation campaign. Here, we consider a dual-deflection mitigation campaign that combines the $\mathrm{KI} / \mathrm{NI} / \mathrm{GT}$ as a primary deflection mission and the GT as a secondary deflection mission in order to overcome the limits imposed on the single asteroid deflection approach and to make the given mitigation campaign more credible. The primary deflection mission makes use of an instantaneous/quasi-instantaneous (e.g. a week/month-long deflection mission by SC) deflection technique whereas the secondary deflection mission makes use of a slow push deflection technique. The final outcome of the given mitigation campaign is therefore determined by the secondary deflection mission which performs its slow-push deflection action according to the instantaneous outcome of the primary deflection mission that could be fully successful, partly successful, or at worst, complete failure. In addition to this, the secondary deflection mission should be capable of preventing the NEA from passing an undesired subsequent Earth impact keyhole on the $2036 \mathrm{~b}$-plane due to the unexpected b-plane deflection of the primary mission.

\subsection{Dual-deflection campaign}

Dual-deflection campaigns studied here consist of a primary KI/NI/SC mission and a secondary GT mission, which are designated as KI-GT, NI-GT, and SC-GT campaigns, respectively. Figure 10 shows an example of KI-GT campaign under the 20-year warning time scenario. The transfer orbits of the KI and GT are determined by solving a conventional two-body Lambert's problem. A conventional chemical propulsion system of $I s p=300$ seconds is assumed as a kick stage at the Earth departure and also at the final approach to the target NEA to accelerate or decelerate. For the case of KI-GT campaign, two mitigation missions are sent to the NEA separately and hence follow two different trajectories. This allows the KI mission to take advantage of a higher relative velocity at the encounter with NEA while allowing the GT mission to rendezvous with the NEA without consuming an excessive amount propellant at NEA arrival. For this reason, the GT's arrival could be earlier than the KI's arrival, in which case the GT will be operational before and after the KI mission. On the other hand, for the case of NI-GT/SC-GT campaign, it is assumed that the two missions are sent together to the target NEA as a single mitigation system stack module while, apart from this difference, all the dual-deflection mitigation campaigns will be designed and optimised in the same manner with respect to the aforementioned series of competing aspects. In any scenario, it is assumed that the GT mission takes place immediately after its arrival at the NEA and is kept in operation regardless of an event associated with the primary deflection mission (e.g. kinetic impact, nuclear blast, solar ablation event, etc.). 


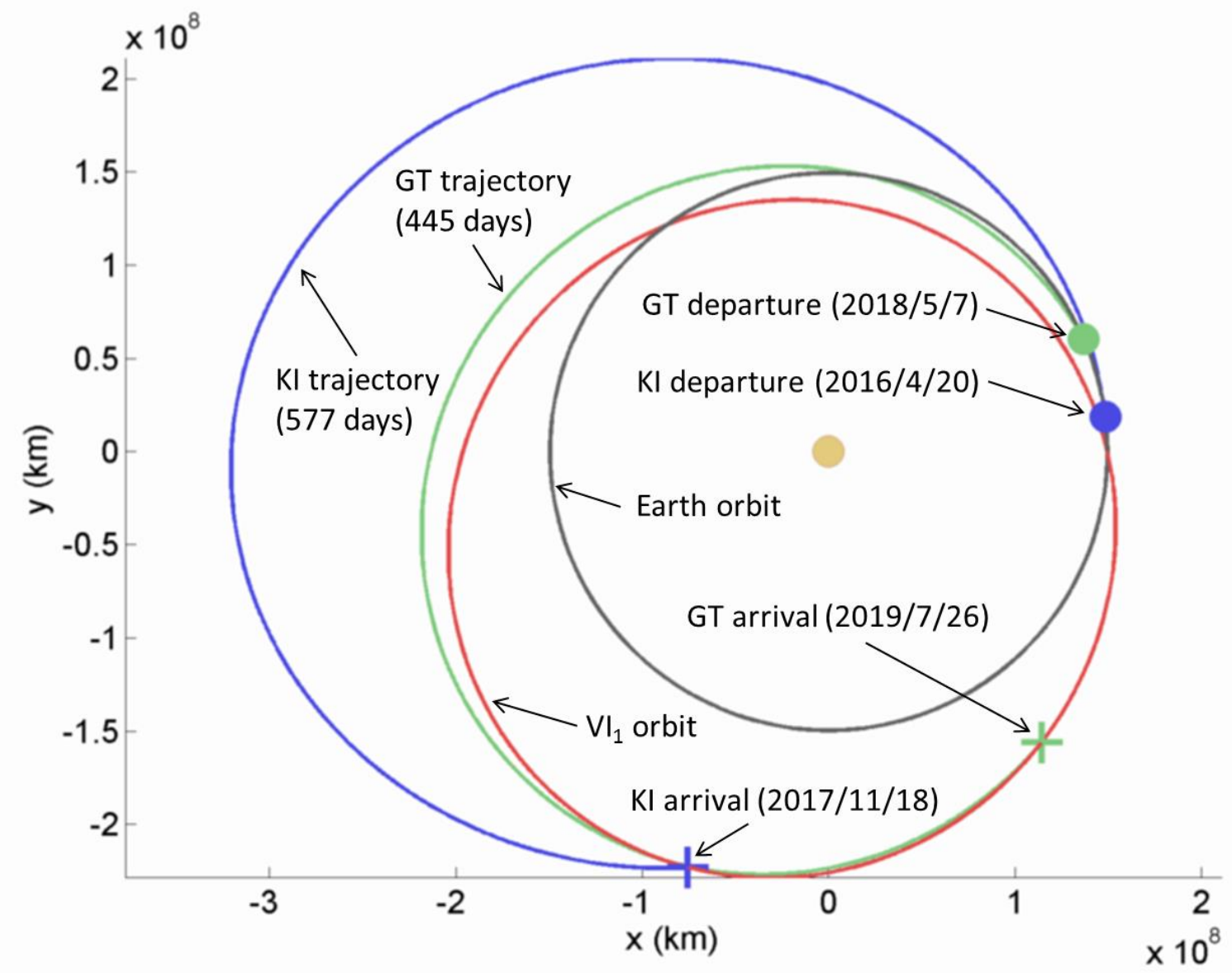

Figure 10 Example of KI-GT campaign. The NEA orbit is represented in red. The transfer orbit of KI is represented in blue. The transfer orbit of GT is represented in green. Earth's orbit is represented in grey. The blue and green circles represent the Earth departure points of the KI and GT, respectively. The KI interception and GT rendezvous points with the NEA are represented as the blue and green cross shapes, respectively.

The system design variables in vector $\mathbf{x}$ and their bounds for KI-GT/NI-GT/SC-GT campaign are shown in Table 10. $m_{1}$ and $m_{2}$ are the masses of primary and secondary mitigation systems at NEA arrival, respectively. $t_{1}$ and $t o f_{1}$ are the Earth departure time and the flight time of the primary deflection mission whereas $t_{2}$ and to $f_{2}$ are the Earth departure time and the flight time of the secondary deflection mission. The latter two are required only for KI-GT campaigns as two deflection missions are independently sent to the asteroid whereas they are not for NI-GT/SC-GT campaigns as two deflection missions are sent together. The primary deflection mission onset time $t_{\text {int1 }}$ must be also defined for the NI-GT/SC-GT campaign as the two mitigation systems will be sent together such that the NEA arrival time may not be always optimal $t_{\mathrm{int} 1}$ of the given primary deflection mission. Note that $\Delta \mathbf{v}_{\text {imp }}\left(=\Delta v_{\text {imp }} \cdot \hat{\mathbf{v}}_{\text {imp }}\right)$ is the impact velocity increment in addition to the terminal NEA encounter relative velocity $\mathbf{v}_{\mathrm{imp}}$ of the impact trajectory of the KI mission. Finally, $\Delta t_{\mathrm{GT}}$ and $\Delta t_{\mathrm{SC}}$ are the time duration of the GT mission and the SC mission, respectively, where $\mathrm{T}_{\mathrm{a}}$ is one orbital period of the given virtual impactor. The maximum value of the SC mission duration $\Delta t_{\mathrm{SC}}$ is therefore one asteroid orbit at most; however, such a long SC mission duration is usually not necessary as will be shown later in this chapter. Interested readers should refer to details about the mass of each mitigation system at the NEA arrival, presented separately in Appendix A.2. 
Table 10 Minimum and maximum values of design variables $\mathbf{x}$ for a KI-GT/NI-GT/SC-GT campaign. The warning time is set to 20 years between 2016 and 2036. $\mathrm{T}_{\mathrm{a}}$ is the orbital period of the target NEA.

\begin{tabular}{llll}
\hline $\mathbf{x}$ & KI-GT & NI-GT & SC-GT \\
\hline$m_{1}(\mathrm{~kg})$ & $100-10000$ & $0-300$ & $0-300$ \\
$m_{2}(\mathrm{~kg})$ & $100-10000$ & $100-10000$ & $100-10000$ \\
$t_{1}$ & $2016 / 4 / 13-2036 / 4 / 13$ & $2016 / 4 / 13-2036 / 4 / 13$ & $2016 / 4 / 13-2036 / 4 / 13$ \\
$t o f_{1}($ day $)$ & $30-1000$ & $30-1000$ & $30-1000$ \\
$t_{2}$ & $2016 / 4 / 13-2036 / 4 / 13$ & & \\
$t o f_{2}($ day $)$ & $30-1000$ & & \\
$t_{\text {int1 }}$ & & $\left(t_{1}+t o f_{1}\right)-t_{\text {MOID }}$ & $\left(t_{1}+\right.$ to $\left._{1}\right)-t_{\text {MOID }}$ \\
$\Delta v_{\text {imp }}(\mathrm{km} / \mathrm{s})$ & $0-100$ & $0-20$ & $0-20$ \\
$\Delta t_{\mathrm{GT}}($ year $)$ & $0-20$ & & $0-1$ \\
$\Delta t_{\mathrm{SC}}\left(\mathrm{T}_{\mathrm{a}}\right)$ & & & \\
\hline
\end{tabular}

\subsection{Campaign optimisation}

The dual-deflection mitigation campaign optimisation problem entails an assessment of the figures of merit (i.e. the mitigation performance indicators) in vector $\mathbf{y}$ that characterise the performance and the confidence in successful deflection through the given mitigation campaign. Seven figures of merit in vector $\mathbf{y}$ are described in Table 11. $m_{0}$ is the total mitigation systems mass at the EDS which is equal to the sum of $m_{\mathrm{EDS} 1}$ and $m_{\mathrm{EDS} 2}$ : the masses of two mitigation systems at the respective Earth departure stages (EDS). $m_{0}$ should be as small as possible to reduce the cost of the mitigation campaign. The upper bound of $m_{0}$ is set to 100 tons. $t_{\mathrm{f} 1}$ and $t_{\mathrm{f} 2}$ are the asteroid arrival time of the mitigation $\operatorname{system}(\mathrm{s})$ and the campaign completion time (i.e. GT termination time), respectively. $t_{\mathrm{f} 1}$ is desirable to be as late as possible such that more time can be spent on decision-making of mitigation campaign enforcement. $t_{\mathrm{f} 2}$, on the other hand, should be as early as possible such that an additional mitigation campaign can be launched, if necessary. Not to mention, $t_{\mathrm{f} 1}$ can be no later than $t_{\mathrm{f} 2}$. $b_{\mathrm{nom}}$ is the nominal deflection on the b-plane and desired to be as large as possible within the range of $1,000 \mathrm{~km} \leq b_{\text {nom }} \leq$ $32,000 \mathrm{~km}$. The lower bound of $b_{\text {nom }}$ refers to the safe amount of deflection (1000 km on the b-plane) that is required to put the b-plane uncertainty ellipsoid off the secondary impact keyholes [26] whereas the upper bound is approximately equal to 5.0 Earth-radii. $B e l_{\text {nom }}$ and $P l_{\text {nom }}$ are the Belief and Plausibility measures of nominal deflection distance. Higher Belief is simply an indication of higher confidence in successful mitigation. Finally, $b_{\text {trim }}$ is the post primary mission trim deflection achieved through the GT mission, which indicates the trim deflection capability of the given dual-deflection mitigation campaign for secondary impact keyhole avoidance. The maximum deflection distance of this trim deflection is set to $1000 \mathrm{~km}$ on the b-plane. 
Table 11 Figures of merit of mitigation campaign for different scenarios: KI-GT, NI-GT, and SC-GT. The smaller these figures are, the more optimal the given mitigation campaign is. Note that $t_{\mathrm{f} 1}$ is to be maximised as a later primary deflection completion time allows the planetary defense framework to delay the decision-making of the campaign as long as possible.

\begin{tabular}{ll}
\hline $\mathbf{y}$ & Description \\
\hline$m_{0}$ & Total mitigation systems mass at EDS $\left(=m_{\mathrm{EDS} 1}+m_{\mathrm{EDS} 2}\right)$ \\
$-t_{\mathrm{f} 1}$ & Primary deflection completion time \\
$t_{\mathrm{f} 2}$ & Secondary deflection completion time \\
$-b_{\text {nom }}$ & Nominal deflection achieved through dual-deflection campaign \\
$-B e l_{\text {nom }}$ & Belief of nominal deflection $b_{\text {nom }}$ \\
$-P l_{\text {nom }}$ & Plausibility of nominal deflection $b_{\text {nom }}$ \\
$-b_{\text {trim }}$ & Trim (post-primary) deflection achieved through by GT mission \\
\hline
\end{tabular}

Minimising the above-mentioned figures of merit inevitably entails a multi-objective optimisation problem. Following the conventional approach of Pareto optimal optimisation [1], the fast and elitist multiobjective genetic algorithm NSGA-II [27] is used to compute Pareto optimal design points of dual-deflection mitigation campaigns. A total of 2400 solutions for $\mathbf{y}$ from a hundred of generations are numerically computed in MATLAB. Detailed information about the multiobjective genetic algorithm is omitted in this paper and thus readers interested in the algorithm should refer to the work of NSGA-II developed by Deb et al. [27].

\section{Results and discussion}

Selected optimisation results (i.e. Pareto-optimal solutions) of the KI-GT, NI-GT, and SC-GT campaigns are presented in the following subsections, respectively.

\subsection{KI-GT campaign}

The Pareto-optimal results of KI-GT campaigns against 140-metre sized S-type $\mathrm{VI}_{1}$ characterised under the ground-based characterisation scenario are shown in Figure 11. The colours of the dots represent the degrees of nominal deflection distances between 0.0-5.0 Earth-radii. The four plots on the left-hand side show the optimal solutions that satisfy $\mathrm{Bel}_{\text {nom }} \geq 0.47$ whereas those on the right-hand side satisfy $\mathrm{Bel}_{\text {nom }}=1.00$. The Pareto-optimal solutions are plotted for: KI Earth departure time vs. total mitigation system mass at EDS ( $t_{1}$ vs. $m_{0}$ ); campaign completion time vs. total mitigation system mass at EDS ( $t_{\mathrm{f} 2}$ vs. $\left.m_{0}\right)$; KI mass vs. GT mass at EDS ( $m_{\mathrm{EDS} 1}$ vs. $\left.m_{\mathrm{EDS} 2}\right)$; and KI Earth departure time vs. post-KI trim deflection, respectively $\left(t_{1}\right.$ vs. $\left.b_{\text {trim }}\right)$.

Figure 11-A) and B) clearly show that many optimal mitigation campaigns are concentrated in the KI Earth departure time $t_{1}$ around 2018. This appears to be due to the amplification effect of the $\mathrm{VI}_{1}$ perihelion passage on asteroid deflection attempts. More importantly, as the Belief of the nominal deflection increases, the number of solutions for the optimal mitigation campaigns decreases, which is simply because a KI-GT campaign with higher degree of Belief requires a larger fraction of the total mitigation system mass $m_{0}$ for the secondary deflection mission (i.e. heavier GT mass at the EDS $m_{\mathrm{EDS} 2}$ for a larger post-KI deflection). In order to allocate a sufficient post-KI deflection through the GT mission, the KI Earth departure time is severely limited to the year 2018's time frame as shown in Figure 11-B). As a consequence, $m_{0}$ becomes substantially heavier than that in the campaigns 
with lower degrees of Belief. This in turn means that, if the campaign credibility is not prioritised, quite a few optimal solutions offer a relatively light-weight (20-40 tons) and late-term KI-GT campaigns (in 2025 or later) which suffice the safe b-plane deflection distance of $2.5 \mathrm{~b}_{\oplus}$ (4.16 Earth-radii) as shown Figure 11-A).

Figure 11-C) shows that there are optimal mitigation campaigns of various nominal deflection distances within a wide range of campaign completion/GT termination time between 2018 and 2036 whereas Figure 11-D) shows that a high confidence mitigation campaign with a high nominal deflection results in a late campaign completion, 2026 or later. However, it can be seen that $t_{\mathrm{f} 2}$ close to 2036 does not necessarily result in an effective mitigation campaign in terms of the nominal deflection distance. This can be interpreted as the inefficiency of GT missions close to the impact/Earth encounter epoch in 2036.

Figure 11-E) and F) show the ratio of KI and GT masses at EDS. It can be seen that dual-deflection mitigation campaigns with large nominal deflection and high confidence level require larger fraction of the total mass $m_{0}$ for the GT mission than the KI mission. This appears to be due to the inefficiency of the GT as a deflection technique with respect to the KI.

When the secondary impact keyhole passage avoidance is concerned, Figure 11-A) and B) show that 2033 will be the last launch opportunity for an effective keyhole mitigation campaign against $\mathrm{VI}_{1}$. Although the solutions around 2033 do not offer a large nominal deflection (dark blue dots), these dual-deflection mitigation campaigns still offer the nominal deflection distance of $>1000 \mathrm{~km}$ which is more than enough to avoid secondary impact keyhole. In addition, the avoidance of undesired keyhole passage due to the primary deflection can be fulfilled, counting on the reserved trim deflection $b_{\text {trim }}$ by means of post-primary deflection GT trim manoeuvre (see Figure 11-G) and H)). Not surprisingly, the earlier the KI interception is, the larger $b_{\text {trim }}$ can be. The periods of time to achieve $b_{\text {trim }}$ range from months to years and are proportional to the nominal deflection and the confidence level/Belief of each campaign.

Especially on the KI-GT campaign scenario against $\mathrm{VI}_{1}$, the GT rendezvous with the NEA could be before/after the KI arrival/interception time depending on the KI-GT campaign's mission sequence. The former case is found to be highly beneficial as it enables proximity characterisation of the NEA as well as the precise guidance of the KI by GT spacecraft. The GT might start operating immediately after the NEA rendezvous before the KI arrival/impact, however most importantly, this is not always the case particularly but subject to the true values of the NEA physical properties. For example, if the in-situ NEA physical characterisation claims that the actual NEA mass is less heavy than the nominal value, the GT mission will simply add an extra deflection to the outcome of the primary deflection.

As a final remark of the KI-GT campaign scenario, in practice, not only the NEA arrival but the Earth departure of the KI could be later than the GT arrival at the NEA, depending on the availability that is subject to the launch window, warning time, NEA orbit, etc. This is actually a critical issue on the KI mitigation system design as primary deflection mission because the GT spacecraft may be able to conduct preliminary characterisation of the NEA at the proximity characterisation level even before the Earth departure of the KI mission; however, the feasibility study of such a precursor characterisation mission by GT followed by a KI mission is out of scope of this paper, hence subject of future work. 
A) $\mathrm{Bel}_{\text {nom }} \geq 0.47$

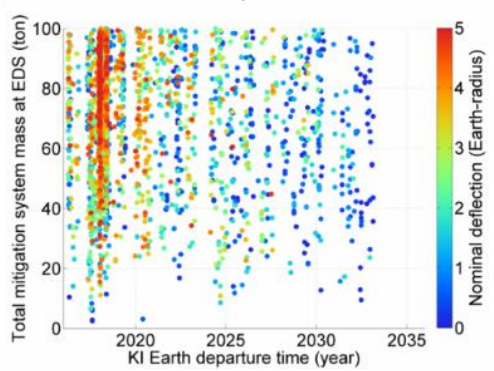

C) Bel $_{\text {nom }} \geq 0.47$

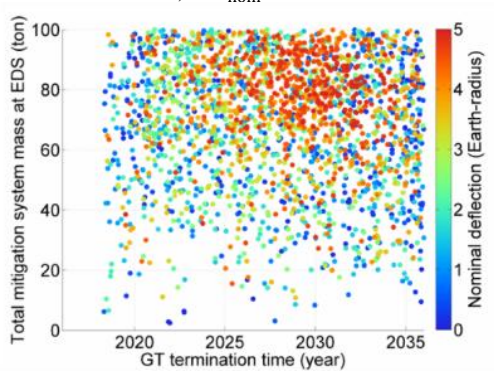

E) Bel $_{\text {nom }} \geq 0.47$

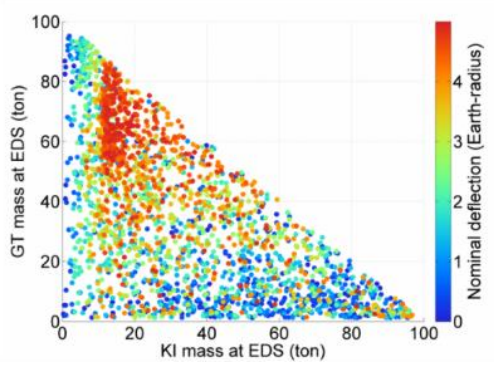

G) Bel $_{\text {nom }} \geq 0.47$

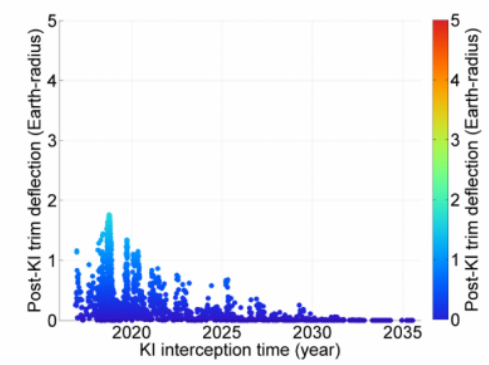

B) $\mathrm{Bel}_{\text {nom }}=1.00$

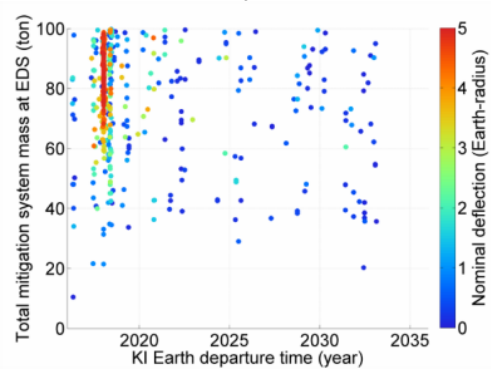

D) $\mathrm{Bel}_{\text {nom }}=1.00$

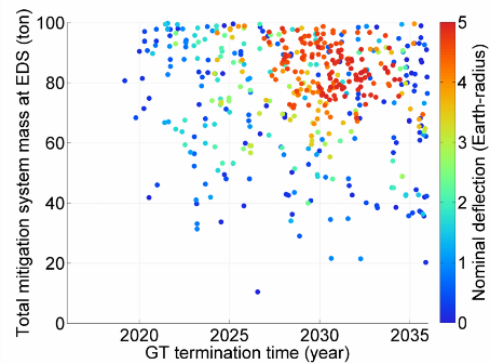

F) $\mathrm{Bel}_{\text {nom }}=1.00$

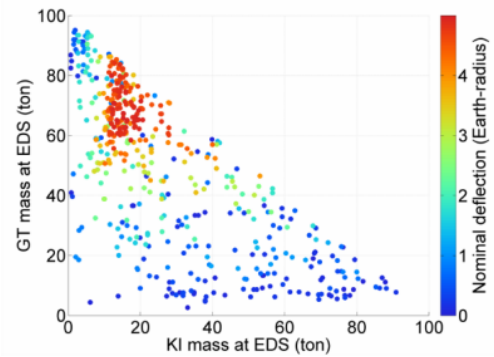

H) $\mathrm{Bel}_{\text {nom }}=1.00$

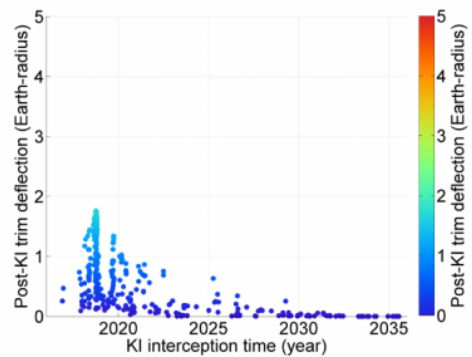

Figure 11 Pareto-optimal solutions for KI-GT campaigns against S-type $\mathrm{VI}_{1}$. The colours of the dots represent the degrees of nominal deflection distances between 0.0-5.0 Earth-radii. The four plots on the left-hand side show the optimal solutions that satisfy $\mathrm{Bel}_{\text {nom }} \geq 0.47$ whereas those on the right-hand side satisfy $\mathrm{Bel}_{\text {nom }}=1.00$.

\subsection{NI-GT campaign}

The results on NI-GT campaigns against 140-metre sized S-type $\mathrm{VI}_{1}$ characterised under the ground-based characterisation scenario are shown in Figure 12 in a similar fashion as the results on KI-GT campaign scenario shown in the previous section.

Figure 12-A) and B) clearly show that the NI-GT mitigation campaign scenario has more distinct optimality in terms of the NI-GT mitigation campaign launch window than the KI-GT mitigation campaign scenario. This might be accounted for by the fact that both NI and GT mitigation systems are delivered to the target asteroid as a single mitigation systems stack and rendezvous with it together such that optimal launch windows are more limited than the KI-GT scenario. As the Belief of the nominal deflection 
increases, the number of solutions for such optimal mitigation campaigns decreases more profoundly than the KI-GT scenario, which is again because of the limited launch opportunities for the mitigation systems stack carrying the two mitigation systems. An NI-GT campaign with higher degree of Belief requires a larger mass for the GT mission while the mass required for the NI mission mostly remains smaller than $300 \mathrm{~kg}$.

A late-stage launch of the mitigation systems stack results in considerable deflection efficiency reduction and eventually regarded as non-optimal by the simulation. For this reason, the mitigation systems' Earth departure time is severely limited particularly when a higher degree of Belief is concerned. On the other hand, if the campaign credibility is not prioritised, quite a few optimal solutions offer a relatively light-weight (10-30 tons) and late-term (2028 ) NI-GT campaigns which suffice the safe b-plane deflection distance of $2.5 \mathrm{~b}_{\oplus}(4.16$ Earth-radii) as shown in Figure 12-A).

Figure 12-C) seems akin to Figure 11-C), showing optimal mitigation campaigns of various nominal deflections within a wide range of campaign completion time between 2018 and 2036. Figure 12-D) is also similar to Figure 11-D), indicating that a mitigation campaign with high Belief and nominal deflection results in a late campaign completion time.

Figure 12-E) and F) show the ratio of NI and GT masses at the NEA arrival instead of those at the EDS because they depart the Earth and sent to the target asteroid together. It can be seen that NI-GT mitigation campaigns basically entail very larger fraction of the total mass for the GT mission than the NI mission. This is simply due to the inefficiency of the GT as a deflection technique with respect to the NI which has the highest deflection yield per unit mass among all the deflection techniques considered in this paper.

Similar to the KI-GT scenario, undesired keyhole passage due to the primary deflection can be avoided, counting on $b_{\text {trim }}$ achieved through the post-NI GT mission (see Figure 12-G) and $\mathrm{H}$ )).

As a final remark of the NI-GT campaign scenario, an NI-GT mitigation campaign generally outperforms a KI-GT mitigation campaign. However, it still requires a relatively heavy GT spacecraft in order to achieve a high Belief/confidence level on the nominal deflection equal to or larger than the safe b-plane deflection. In reality, the NI and GT deflection missions will take place after the target asteroid is carefully characterised by spacecraft. This will substantially reduce the epistemic uncertainties in the fundamental NEA characteristics such as the mass, density, porosity, shape, etc. If that is the case, the NI mission can refer to the actual physical properties of the target body and regulate the NI deflection yield by changing the stand-off distance such that the nominal deflection can be achieved without the help of the post-NI deflection by GT mission for instance. 
A) Bel $_{\text {nom }} \geq 0.47$

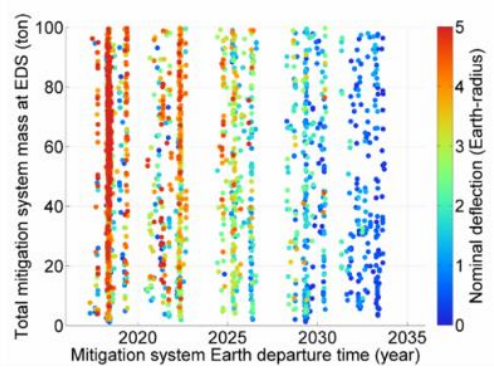

C) $\mathrm{Bel}_{\text {nom }} \geq 0.47$

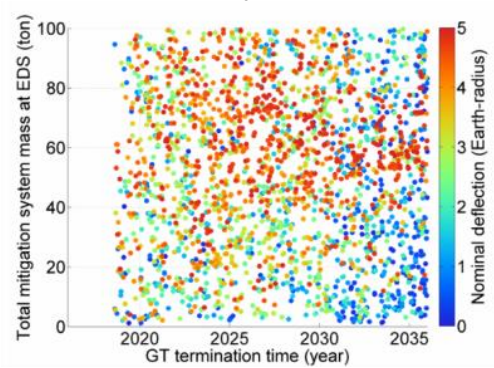

E) $\mathrm{Bel}_{\text {nom }} \geq 0.47$

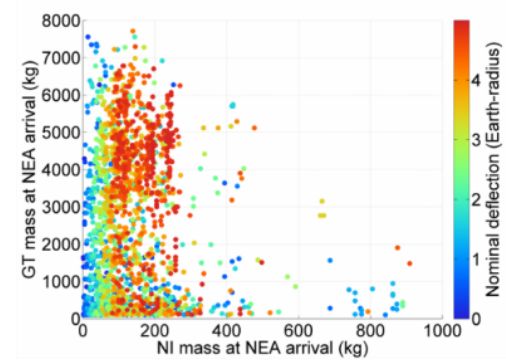

G) Bel $_{\text {nom }} \geq 0.47$

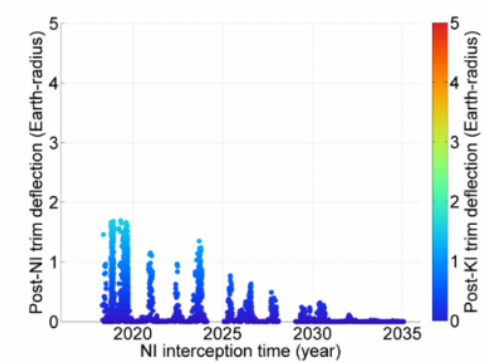

B) $\mathrm{Bel}_{\text {nom }}=1.00$

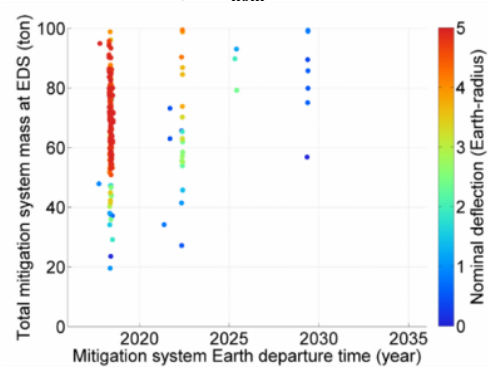

D) $\mathrm{Bel}_{\text {nom }}=1.00$

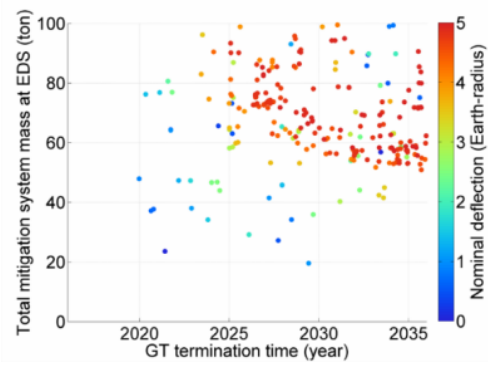

F) $\mathrm{Bel}_{\text {nom }}=1.00$

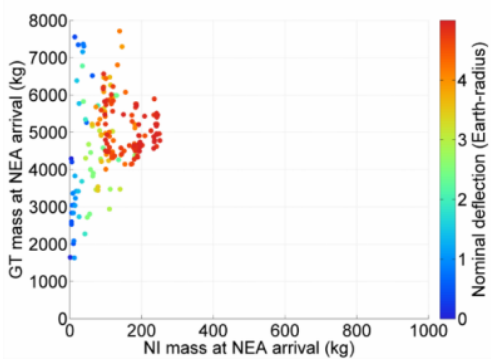

H) $\mathrm{Bel}_{\text {nom }}=1.00$

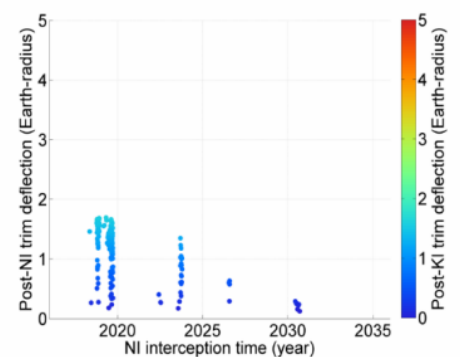

Figure 12 Pareto-optimal solutions for NI-GT campaigns against S-type $\mathrm{VI}_{1}$. The colours of the dots represent the degrees of nominal deflection distances between 0.0-5.0 Earth-radii. The four plots on the left-hand side show the optimal solutions that satisfy $\mathrm{Bel}_{\text {nom }} \geq 0.47$ whereas those on the right-hand side satisfy $\mathrm{Bel}_{\text {nom }}=1.00$.

\subsection{SC-GT campaign}

The results of SC-GT campaigns against 140-metre sized S-type $\mathrm{VI}_{1}$ characterised under the ground-based characterisation scenario are shown in Figure 13 in the same fashion as the results on NI-GT campaign scenario. Figure 13-A) and B) show that there exist most of the optimal mitigation campaigns with large nominal deflections (i.e. red dots) having the SC Earth departure time $t_{1}$ around 2018 while those campaigns after 2018 become much less efficient in terms of nominal deflection leverage. Particularly when a higher value of Belief measure is concerned, the availability of mitigation campaigns with reasonable deflection after 2018 is strictly limited. According to Figure 13-G) and H), these optimal mitigation campaigns available around 2018 are associated with a relatively large fraction of the resultant b-plane deflection achieved through the GT mission. This be interpreted as the considerably large effects of the epistemic uncertainties in NEA physical properties on 
the SC deflection efficiency. On the other hand, if the campaign credibility is not prioritised, some optimal solutions after 2025 offer a relatively light-weight (20-40 tons) SC-GT campaign which suffices the safe b-plane deflection distance of $2.5 \mathrm{~b}_{\oplus}$ (4.16 Earth-radii) as can be seen in Figure 13-A).

Similar to the KI-GT and NI-GT scenarios, Figure 13-C) and D) show that there are optimal campaigns of various nominal deflection distances within a wide range of campaign completion time between 2020 and 2036. The optimal SC-GT mitigation campaign with the earliest completion time can be found around 2020 which is approximately two years later than those for the KI-GT and NI-GT mitigation campaign scenarios around 2018. This can be accounted for by the fact that the SC mission takes a few weeks to months in order to complete its deflection attempt.

Figure 13-E) and F) show the ratio of SC and GT masses at NEA arrival. It can be seen that SC-GT mitigation campaigns generally require larger fraction of the total mitigation system mass for the GT mission than for the SC mission. This is due to the inefficiency of the GT as a deflection technique in comparison with the SC which is one of the most optimal deflection technique among the four deflection techniques considered in this doctoral paper. If a higher value of Belief measure is concerned, the GT mass becomes substantially larger than the SC mass. This is again due to the inefficiency of the GT as an asteroid deflection technique as well as due to the large uncertain outcome of the SC mission. It is therefore highly desirable to improve the deflection efficiency of the GT mission or simply to send more than a single SC mission to the target asteroid for the SC-GT campaign scenario.

When the secondary impact keyhole passage is concerned, Figure 13-A) and B) show that 2029 will be basically the last launch opportunity for an effective mitigation campaign against $\mathrm{VI}_{1}$. In addition, the avoidance of undesired keyhole passage due to the primary deflection can be fulfilled, counting on the reserved deflection $b_{\text {trim }}$ by post-SC deflection achieved through the GT mission (see Figure 13-G) and $\mathrm{H}$ )). It seems that large $b_{\text {trim }}$ is provided through the GT mission when the SC mission takes place in 2019 and thus the mitigation campaigns are more efficient than the late-term ones. 
A) Bel $_{\text {nom }} \geq 0.47$

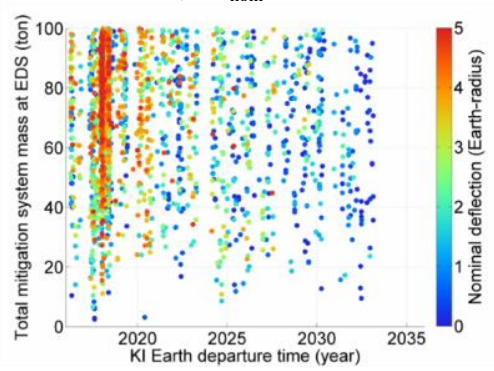

C) $\mathrm{Bel}_{\text {nom }} \geq 0.47$

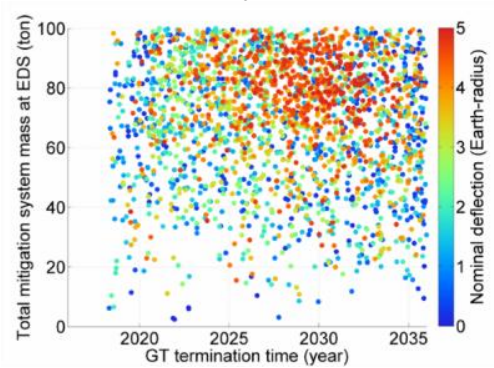

E) Bel $_{\text {nom }} \geq 0.47$

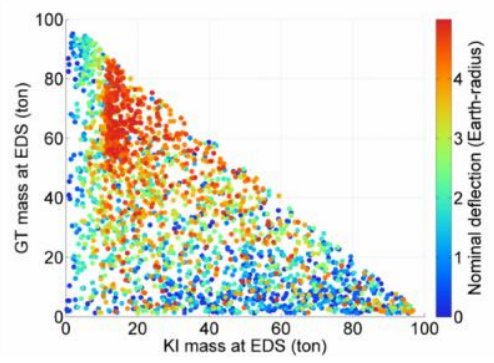

G) Bel $_{\text {nom }} \geq 0.47$

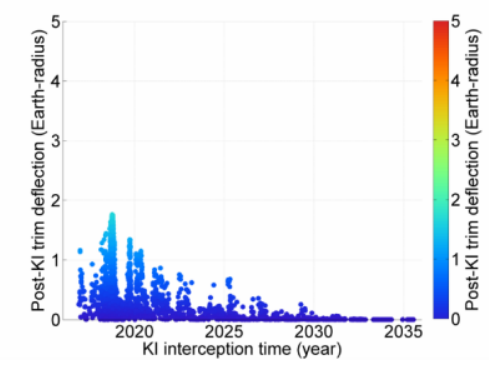

B) $\mathrm{Bel}_{\text {nom }}=1.00$

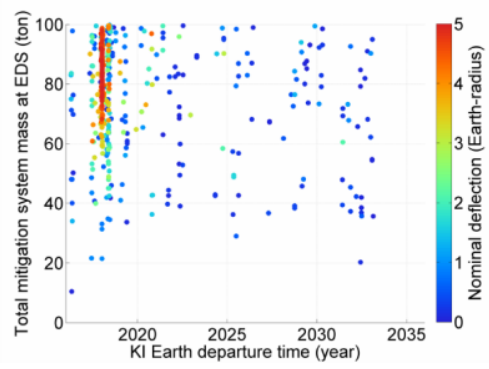

D) $\mathrm{Bel}_{\text {nom }}=1.00$

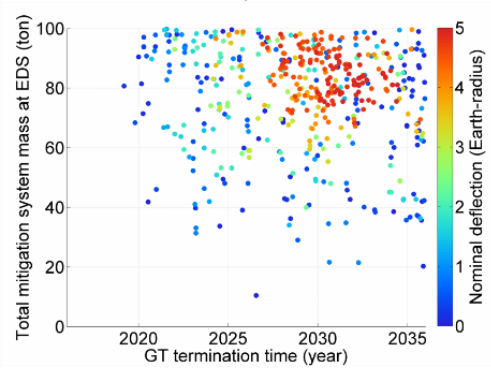

F) $\mathrm{Bel}_{\text {nom }}=1.00$

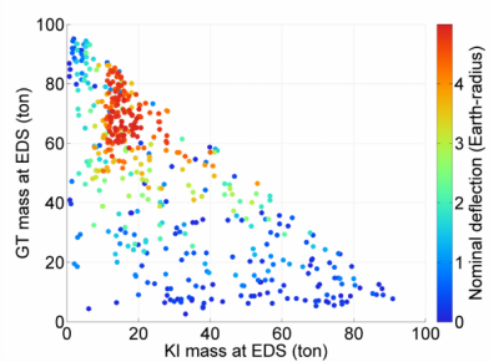

H) $\mathrm{Bel}_{\text {nom }}=1.00$

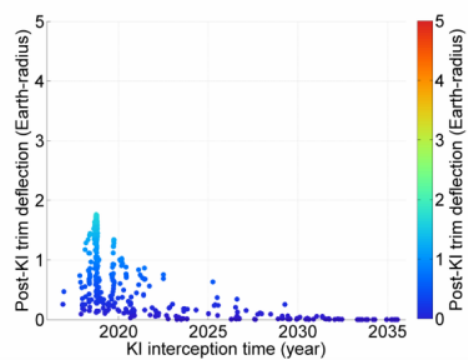

Figure 13 Pareto-optimal solutions for SC-GT campaigns against S-type $\mathrm{VI}_{1}$. The colours of the dots represent the degrees of nominal deflection distances between 0.0-5.0 Earth-radii. The four plots on the left-hand side show the optimal solutions that satisfy $\mathrm{Bel}_{\text {nom }} \geq 0.47$ whereas those on the right-hand side satisfy $\mathrm{Bel}_{\text {nom }}=1.00$.

\section{Conclusions}

Hazardous NEA mitigation campaign planning under the uncertainty-based information on the fundamental asteroid characteristics has been studied to improve the mitigation campaign credibility, where one of the possible forms of mitigation campaign - dual-deflection campaign - has been investigated in detail. In order to evaluate the confidence level of deflection missions subject to the uncertain NEA characteristics, the uncertainty quantification technique called Evidence Theory is used. The preliminary results of the dual-deflection campaigns consisting of a primary $\mathrm{KI} / \mathrm{NI} / \mathrm{SC}$ and a secondary GT have shown that: 
- Dual-deflection campaign planning involves a series of competing aspects that must be assessed and constraints associated with the specific configurations of KI-GT/NI-GT/SC-GT to be satisfied to plan a mitigation campaign with sufficient performance (i.e. deflection) and high confidence in successful deflection (i.e. Belief).

- Given a GT as a secondary deflection mission, the Belief of nominal deflection can be improved by years of orbit trim manoeuvre by GT before and after a primary deflection mission for the dual-deflection scenario. However, this does not necessarily means that the GT must always commence its deflection action immediately after its NEA rendezvous but the actual operation of the GT is subject to the in-situ NEA characteristics as well as the outcome of primary deflection mission.

- Given 10-20 years of warning time, a mitigation campaign with a completion time of approximately half the warning time seems to be more reasonable than a longer-term mitigation campaign or a mitigation campaign with a heavier total mitigation systems mass at the EDS. This appears to be due to the deflection efficiency decrease with time and the high launch cost of necessary mitigation systems for any late-term mitigation campaigns.

- Possible keyhole passage due to undesired deflection by primary KI/NI/SC mission can be avoided by GT as a secondary deflection mission in a dual-deflection campaign, given the necessary amount of deflection in order to avoid the keyhole passage is $1000 \mathrm{~km}$.

Finally, the particular campaign planning approach presented here could be useful for the near-future hazard mitigation campaigns where we might have to tap into our incomplete knowledge of NEAs for mitigation campaign design, allowing to select the best possible combination of deflection missions from a catalogue of various possible mitigation campaign options, without compromising the campaign credibility. However, in the foreseeable future, further knowledge about the NEA population and some specific NEAs will have steadily accumulated and improved through the forthcoming NEA survey and exploration missions such as NEOSSat, Sentinel, Hayabusa2, and OSIRIS-Rex as well as the recently announced NASA's NEA retrieval mission to be launched as soon as 2017.

\section{Acknowledgement}

The authors would like to thank the Committee of the 2013 IAA Planetary Defense Conference for offering the opportunity to publish this work in the special edition of Acta Astronautica. 


\section{Appendices}

A.1. BPA structures for NEA physical properties

The uncertain NEA physical properties, more specifically, micro density $\rho_{\text {micro }}$, micro porosity $P_{\text {micro }}$, bulk porosity $P_{\text {bulk }}$, and albedo $p_{v}$ are represented in the forms of BPA structures (i.e. a set of interval values with prescribed BPAs), respectively. The BPA structures for the S-type, C-type, and M-type NEAs are given in Table 12. Also, three different characterisation scenarios (the ground-based, space-based, and proximity-based characterisation scenarios) are considered, where differences in uncertainty level are represented appropriately by scaling down the overall interval of any parameter which is eligible to be more certain and by referring to the capability and limits of respective characterisation scenarios. The following sub-subsections present the details on the formation of the BPA structures for the respective taxonomic classes and the characterisation scenarios, acknowledging all the corresponding supporting information regarding these NEA physical properties.

\section{A.1.1. S-type}

The meteorite analogues of S-type asteroids were believed to be stony meteorites even before the Hayabusa spacecraft visited the S-type asteroid, namely 25143 Itokawa. The Hayabusa mission finally confirmed directly that the characteristics of Itokawa's surface grains returned by the Hayabusa spacecraft are consistent with the characteristics of L, LL, and partly $\mathrm{H}$ chondrites [28]. Most importantly, the Hayabusa mission has proved the analogy between meteorites and NEA compositions through the in-situ study of the asteroid for the first time.

The microscopic physical parameters of S-type asteroids are often estimated by referring to their meteorite analogues - ordinary chondrites (OCs), assuming they are homogeneously composed of OCs, L, LL, and H chondrites in particular. Although, the Hayabusa mission revealed that Itokawa's surface is mostly made of L and LL chondrites [29], we cannot rule out the possible presence of subsurface metal-rich particles such as $\mathrm{H}$ chondrites buried inside Itokawa due to successive impact events.

BPA structures for the physical properties of S-type asteroids are given in Table 12. The BPA structures for the micro density $\rho_{\text {micro }}$ are formed by referring to 437 samples of $\mathrm{H}, \mathrm{L}$, and LL chondrites [30]. In order to form the BPA structures for the micro porosity $P_{\text {micro }}$, micro porosities of 691 OCs [31] are referred and aggregated with additional data on micro porosities of 291 OCs from the different literature [32] by Dempster's rule of combination [22]. While most meteorites found on Earth spent long periods of time on the ground, and thus they are affected by considerable terrestrial weathering, it is noteworthy that the literature referred for $P_{\text {micro }}$ of OCs used in this study is de-biased to such effects. This is due to the fact that the majority of meteorite falls are stony meteorites and about $80 \%$ of which are OCs, hence the amount of information on non-weathered OCs is consequently most abundant among our collection of meteorite collection.

The abundance of S-type asteroids amongst the well-characterised asteroids [16] and the series of exploration missions to the S-type asteroids also results in better understanding of the macroscopic characteristics of S-type asteroids. The BPA structures for the bulk porosity $P_{\text {bulk }}$ of S-type asteroids are formed by referring to the bulk porosities of 7 existing S-type asteroids (smaller than $100 \mathrm{~km}$ in diameter) with bulk porosities ranging 20-60\% [16]. Finally, the BPA structures for the albedo $p_{v}$ are formed by referring to the observed geometric albedos of 30 different S-type asteroids [33]. 


\section{A.1.2. C-type}

The microscopic physical parameters of C-type asteroids can be roughly estimated by referring to their meteorite analogues - carbonaceous chondrites (CCs), assuming they are homogeneously composed of CCs. Since CCs represent less than $5 \%$ of stony meteorite falls, the information on non-weathered CCs is limited.

BPA structures for the physical properties of C-type asteroids are given in Table 12. The BPA structures for the micro density $\rho_{\text {micro }}$ is formed by referring to $11 \mathrm{CM}$ chondrites [34]. The micro porosity $P_{\text {micro }}$ is simply assumed to be 15.5-30.5\% [30] which is consistent with the work of Flynn et al. [35]. This simple assumption is mainly due to the insufficient amount of sample data for unbiased micro porosities of CCs.

C-type asteroids are second most abundant amongst the discovered NEAs, however unlike S-type asteroids, in-situ exploration missions to C-type asteroids (e.g. Hayabusa 2 and OSIRIS-REx) are yet to be sent, and hence the macroscopic characteristics of C-type asteroids are highly unknown. The BPA structures for the bulk porosity $P_{\text {bulk }}$ of C-type asteroids are formed by referring to 8 existing C-type asteroids of various sizes with bulk porosities ranging 30-70\% [16]. Finally, the BPA structures for the albedo $p_{v}$ are formed by referring to the observed geometric albedos of 18 different C-type asteroids [33].

\section{A.1.3. M-type}

Some of the discovered M-type asteroids (e.g. 21 Lutetia, 22 Kalliope, 129 Antigone, and 785 Zwetana) are not entirely consistent with typical iron-nickel meteorite analogues whereas (e.g. 16 Psyche, 216 Klepatra, and (6178) 1986 DA) appear to be metallic. One of the smallest M-type asteroids, namely (6178) 1986 DA is a very good candidate of metallic asteroids which are thought to be a remnant of the core of a fractured primitive body from the early solar system. The meteorite analogues of metallic M-type asteroids are basically iron meteorites.

The microscopic physical parameters of M-type asteroids can be roughly estimated by referring to their meteorite analogues - iron meteorites, assuming they are homogeneously composed of iron meteorites. Iron meteorites represent less than 6\% [36] of all the meteorite falls, and thus the amount of information on non-weathered iron meteorites is most limited.

Iron meteorites are known to be more immune to terrestrial weathering, and thus there is usually less difference between weathered and unweathered samples than fragile chondrite meteorites. BPA structures for the physical properties of M-type asteroids are given in Table 12. The BPA structures for the micro density $\rho_{\text {micro }}$ is formed by referring to 21 iron-nickel meteorites [37]. The micro porosity $P_{\text {micro }}$ is simply assumed to be $0-1.2 \%$ [38]. This range is consistent with the iron-nickel meteorites of the Vatican collection with porosities of near zero [39].

M-type asteroids are least abundant amongst the three asteroid types, and thus their macroscopic characteristics are mostly unknown. Not to mention, they have not been characterised by spacecraft and, unlike S-type and C-type asteroids, remote characterisation of M-type asteroids is not an easy task because the spectrum analysis of them provide us with less information than that of the other two types does. The BPA structures for the bulk porosity $P_{\text {bulk }}$ of M-type asteroids are formed by referring to 7 existing M-type asteroids of various sizes with bulk porosities ranging $0-40 \%$ [16]. Finally, the BPA structures for the albedo $p_{v}$ are formed by referring to the observed geometric albedo of 10 different M-type asteroids [33]. 
Table 12 BPA structures for the fundamental NEA physical properties of S-type, C-type, and M-type asteroids.

A) Ground-based characterisation. B) Space-based characterisation. C) Proximity characterisation.

\begin{tabular}{|c|c|c|c|c|c|c|}
\hline \multirow[t]{2}{*}{ A) } & \multicolumn{2}{|l|}{ S-type } & \multicolumn{2}{|l|}{ C-type } & \multicolumn{2}{|l|}{ M-type } \\
\hline & Interval & BPA & Interval & BPA & Interval & BPA \\
\hline \multirow{4}{*}{$\rho_{\text {micro }}\left(\mathrm{g} / \mathrm{cm}^{3}\right)$} & {$[3.23,3.30]$} & 0.31 & {$[2.57,2.60]$} & 0.18 & {$[7.59,7.60]$} & 0.05 \\
\hline & {$[3.30,3.50]$} & 0.29 & {$[2.60,2.70]$} & 0.36 & {$[7.60,7.80]$} & 0.19 \\
\hline & {$[3.50,3.70]$} & 0.33 & {$[2.70,2.80]$} & 0.18 & {$[7.80,8.00]$} & 0.62 \\
\hline & {$[3.70,3.84]$} & 0.07 & {$[2.80,2.86]$} & 0.27 & {$[8.00,8.07]$} & 0.14 \\
\hline \multirow[t]{6}{*}{$P_{\text {micro }}(\%)$} & {$[3.7,5.0]$} & 0.01 & {$[15.5,30.5]$} & 1.00 & {$[0.0,1.2]$} & 1.00 \\
\hline & {$[5.0,7.5]$} & 0.10 & & & & \\
\hline & {$[7.5,10.0]$} & 0.30 & & & & \\
\hline & {$[10.0,12.5]$} & 0.32 & & & & \\
\hline & {$[12.5,15.0]$} & 0.22 & & & & \\
\hline & {$[15.0,16.3]$} & 0.05 & & & & \\
\hline \multirow{4}{*}{$P_{\text {bulk }}(\%)$} & {$[16.4,30.0]$} & 0.43 & {$[27.5,40.0]$} & 0.25 & {$[0.0,10.0]$} & 0.43 \\
\hline & {$[30.0,40.0]$} & 0.14 & {$[40.0,50.0]$} & 0.37 & {$[10.0,20.0]$} & 0.14 \\
\hline & {$[40.0,50.0]$} & 0.29 & {$[50.0,60.0]$} & 0.25 & {$[20.0,30.0]$} & 0.14 \\
\hline & {$[50.0,56.0]$} & 0.14 & {$[60.0,67.8]$} & 0.13 & {$[30.0,37.9]$} & 0.29 \\
\hline \multirow{4}{*}{$p_{v}$} & {$[0.10,0.15]$} & 0.27 & {$[0.04,0.05]$} & 0.33 & {$[0.08,0.11]$} & 0.30 \\
\hline & {$[0.15,0.20]$} & 0.37 & {$[0.05,0.06]$} & 0.44 & {$[0.11,0.13]$} & 0.40 \\
\hline & {$[0.20,0.25]$} & 0.23 & {$[0.06,0.07]$} & 0.17 & {$[0.13,0.16]$} & 0.10 \\
\hline & {$[0.25,0.28]$} & 0.13 & {$[0.07,0.073]$} & 0.06 & {$[0.16,0.17]$} & 0.20 \\
\hline \multirow[t]{2}{*}{ B) } & S-type & & C-type & & M-type & \\
\hline & Interval & BPA & Interval & BPA & Interval & BPA \\
\hline \multirow[t]{4}{*}{$\rho_{\text {micro }}\left(\mathrm{g} / \mathrm{cm}^{3}\right)$} & {$[3.23,3.30]$} & 0.31 & {$[2.57,2.60]$} & 0.18 & {$[7.59,7.60]$} & 0.05 \\
\hline & {$[3.30,3.50]$} & 0.29 & {$[2.60,2.70]$} & 0.36 & {$[7.60,7.80]$} & 0.19 \\
\hline & {$[3.50,3.70]$} & 0.33 & {$[2.70,2.80]$} & 0.18 & {$[7.80,8.00]$} & 0.62 \\
\hline & {$[3.70,3.84]$} & 0.07 & {$[2.80,2.86]$} & 0.27 & {$[8.00,8.07]$} & 0.14 \\
\hline \multirow[t]{6}{*}{$P_{\text {micro }}(\%)$} & {$[3.7,5.0]$} & 0.01 & {$[15.5,30.5]$} & 1.00 & {$[0.0,1.2]$} & 1.00 \\
\hline & {$[5.0,7.5]$} & 0.10 & & & & \\
\hline & {$[7.5,10.0]$} & 0.30 & & & & \\
\hline & {$[10.0,12.5]$} & 0.32 & & & & \\
\hline & {$[12.5,15.0]$} & 0.22 & & & & \\
\hline & {$[15.0,16.3]$} & 0.05 & & & & \\
\hline \multirow{4}{*}{$P_{\text {bulk }}(\%)$} & {$[21.4,30.0]$} & 0.43 & {$[32.5,40.0]$} & 0.25 & {$[2.86,10.0]$} & 0.43 \\
\hline & {$[30.0,40.0]$} & 0.14 & {$[40.0,50.0]$} & 0.37 & {$[10.0,20.0]$} & 0.14 \\
\hline & {$[40.0,50.0]$} & 0.29 & {$[50.0,60.0]$} & 0.25 & {$[20.0,30.0]$} & 0.14 \\
\hline & {$[50.0,51.4]$} & 0.14 & {$[60.0,62.5]$} & 0.13 & {$[30.0,32.9]$} & 0.29 \\
\hline \multirow[t]{4}{*}{$p_{v}$} & {$[0.14,0.15]$} & 0.31 & {$[0.041,0.05]$} & 0.33 & {$[0.09,0.11]$} & 0.30 \\
\hline & {$[0.15,0.20]$} & 0.42 & {$[0.05,0.06]$} & 0.44 & {$[0.11,0.13]$} & 0.40 \\
\hline & {$[0.20,0.25]$} & 0.27 & {$[0.06,0.07]$} & 0.17 & {$[0.13,0.15]$} & 0.10 \\
\hline & & & {$[0.07,0.071]$} & 0.06 & {$[0.15,0.16]$} & 0.20 \\
\hline \multirow[t]{2}{*}{ C) } & S-type & & C-type & & M-type & \\
\hline & Interval & BPA & Interval & BPA & Interval & BPA \\
\hline \multirow[t]{4}{*}{$\rho_{\text {micro }}\left(\mathrm{g} / \mathrm{cm}^{3}\right)$} & {$[3.26,3.30]$} & 0.31 & {$[2.59,2.60]$} & 0.18 & {$[7.64,7.80]$} & 0.20 \\
\hline & {$[3.30,3.50]$} & 0.29 & {$[2.60,2.70]$} & 0.36 & {$[7.80,8.00]$} & 0.65 \\
\hline & {$[3.50,3.70]$} & 0.33 & {$[2.70,2.80]$} & 0.18 & {$[8.00,8.04]$} & 0.15 \\
\hline & {$[3.70,3.76]$} & 0.07 & {$[2.80,2.83]$} & 0.27 & & \\
\hline \multirow[t]{3}{*}{$P_{\text {micro }}(\%)$} & {$[8.2,10.0]$} & 0.35 & {$[19.8,26.3]$} & 1.00 & {$[0.0,1.2]$} & 1.00 \\
\hline & {$[10.0,12.5]$} & 0.39 & & & & \\
\hline & {$[12.5,13.2]$} & 0.26 & & & & \\
\hline$P_{\text {bulk }}(\%)$ & {$[34.4,34.4]$} & 1.00 & {$[45.7,45.7]$} & 1.00 & {$[13.1,13.1]$} & 1.00 \\
\hline \multirow[t]{2}{*}{$p_{v}$} & {$[0.17,0.20]$} & 0.61 & {$[0.049,0.05]$} & 0.43 & {$[0.11,0.13]$} & 0.80 \\
\hline & {$[0.20,0.21]$} & 0.39 & {$[0.05,0.06]$} & 0.57 & {$[0.13,0.131]$} & 0.20 \\
\hline
\end{tabular}


The mass of each mitigation system at the NEA arrival (regardless of its type: high-velocity interception or rendezvous) is briefly explained here.

The mass of the KI system $m_{\mathrm{KI}}$ is given by Equation (15). $m_{\mathrm{imp}}$ is the fraction of the KI system that actually makes an impact with the asteroid and $\left.m_{\text {prop }}\right|_{\mathrm{TG}}$ is the propellant mass for terminal guidance of the KI system. $m_{\mathrm{imp}}$ is therefore basically the dry mass of the KI system. In this paper, terminal guidance control itself is not analysed in detail and this propellant accounts for the KI system's terminal acceleration through the chemical thruster before the very moment of the interception.

$$
m_{\mathrm{KI}}=m_{\mathrm{imp}}+\left.m_{\mathrm{prop}}\right|_{\mathrm{TG}}
$$

The mass of the NI system $m_{\mathrm{NI}}$ is given by Equation (16), where it is assumed that a $30 \%$ fraction of $m_{\mathrm{NI}}$ accounts for the mass of the nuclear warhead $m_{\mathrm{WH}}$ onboard the NI system and the rest accounts for the mass of all the necessary components for a deep space exploration probe like Deep Space 1 or Hayabusa [40, 41].

$$
m_{\mathrm{NI}}=10 / 3 m_{\mathrm{WH}}
$$

The mass of the SC spacecraft $m_{\mathrm{SC}}$ is given by Equation (17) as a function of the collector's diameter $d_{\mathrm{SC}}$ by reference to the system mass breakdown and model for the solar concentrator "umbrella" from the work of Gritzner and Kahle [42]. It is assumed that $30 \%$ of $m_{\mathrm{SC}}$ accounts for the SC assembly [43] and the other $70 \%$ of $m_{\mathrm{SC}}$ accounts for the thruster/beam generator, power subsystem, propulsion system, propellant masses, etc. The first term of Equation (18) accounts for the mass of the collector's aluminium-coated $90 \%$ reflectivity Mylar (18.9 $\left.\mathrm{g} / \mathrm{m}^{2}\right)$. The second term accounts for the mass of telescopic rods $(2.4 \mathrm{~kg} / \mathrm{m})$, central boom $(0.5 \mathrm{~kg} / \mathrm{m})$, and ropes $(0.16 \mathrm{~kg} / \mathrm{m})$. The last one represents the total mass of eight $4-\mathrm{kg}$ winches. Readers interested in more details on the configuration of the "umbrella" concept should refer to the previous literature [42].

$$
m_{\mathrm{SC}}=10 / 3 m_{\mathrm{CA}}=10 / 3\left(18.9 \times 10^{-3} \cdot \pi \cdot\left(\frac{d_{\mathrm{SC}}}{2}\right)^{2}+3.06 d_{\mathrm{SC}}+32\right)
$$

Finally, the mass of the GT spacecraft $m_{\mathrm{GT}}$ is the sum of the dry mass $m_{\mathrm{dry}}$ and the propellant mass for hovering. It is assumed that $50 \%$ of the dry mass accounts for the mass of power supply $m_{\mathrm{ps}}$ [43].

$$
\begin{gathered}
m_{\mathrm{GT}}=m_{\mathrm{dry}}+\left.m_{\mathrm{prop}}\right|_{\text {hover }} \\
m_{\mathrm{ps}}=0.5 m_{\mathrm{dry}}
\end{gathered}
$$




\section{References}

[1] J.P. Sanchez, C. Colombo, M. Vasile, G. Radice, Multicriteria Comparison Among Several Mitigation Strategies for Dangerous Near-Earth Objects, Journal of Guidance, Control, and Dynamics, 32 (2009) pp. 121-142.

[2] Defending Planet Earth: Near-Earth Object Surveys and Hazard Mitigation Strategies: Final Report, in, Committee to Review Near-Earth Object Surveys and Hazard Mitigation Strategies; National Research Council, 2010.

[3] D. Morrison, C.R. Chapman, D. Steel, R.P. Binzel, Impacts and the public: communicating the nature of the impact hazard, in: M.J.S. Belton, T.H. Morgan, N.H. Samarasinha, D.K. Yeomans (Eds.) Mitigation of Hazardous Comets and Asteroids, Cambridge University Press, Cambridge, 2004, pp. 353-390.

[4] R.B. Adams, J.W. Campbell, R.C. Hopkins, W.S. Smith, W. Arnold, M. Baysinger, T. Crane, P. Capizzo, S. Sutherlin, J. Dankanich, G. Woodcock, G. Edlin, J. Rushing, L. Fabisinski, D. Jones, S. McKamey, S. Thomas, C. Maccone, G. Matloff, J. Remo, Near Earth Object (NEO) Mitigation Options Using Exploration Technologies, in: 2007 Planetary Defense Conference, Washington, DC, 2007.

[5] Y. Sugimoto, G. Radice, J.P. Sanchez, Effects of NEO composition on deflection methodologies, Acta Astronautica, in press (2012) DOI:10.1016/j.actaastro.2012.1008.1030.

[6] A. Milani, S.R. Chesley, P.W. Chodas, G.B. Valsecchi, Asteroid Close Approaches: Analysis and Potential Impact Detection, in: W.F. Bottke Jr., A. Cellino, P. Paolicchi, R.P. Binzel (Eds.) Asteroids III, University of Arizona Press, Tucson, 2002, pp. 55-69.

[7] Near-Earth Object (NEO) analysis of transponder tracking and gravity tractor performance, in, JPL Task Plan No. 82-120022, 2008.

[8] A.W. Harris, M.A. Barucci, J.L. Cano, A. Fitzsimmons, M. Fulchignoni, S.F. Green, D. Hestroffer, V. Lappas, W. Lork, P. Michel, D. Morrison, D. Payson, F. Schaefer, A global approach to near-Earth object impact threat mitigation, in: 2011 IAA Planetary Defense Conference, International Academy of Astronautics, Bucharest, 2011.

[9] S. Hasegawa, T.G. Müller, K. Kawakami, T. Kasuga, T. Wada, Y. Ita, N. Takato, H. Terada, T. Fujiyoshi, M. Abe, Albedo, Size, and Surface Characteristics of Hayabusa-2 Sample-Return Target 1621731999 JU3 from AKARI and Subaru Observations, Publications of the Astronomical Society of Japan, 60 (2008) pp. S399-S405. [10] R.B. Adams, J.W. Campbell, R.C. Hopkins, W.S. Smith, W. Arnold, J. Sverdrup, M. Baysinger, T. Crane, P. Capizzo, S. Sutherlin, J. Dankanich, G. Woodcock, G. Edlin, J. Rushing, L. Fabisinski, D. Jones, S. McKamey, S. Thomas, C. Maccone, G. Matloff, J. Remo, Near Earth Object (NEO) Mitigation Options Using Exploration Technologies, in: Planetary Defense Conference, Washington, DC, 2007.

[11] P. Michel, Physical properties of Near-Earth Objects that inform mitigation, Acta Astronautica, (2012) DOI:10.1016/j.actaastro.2012.1007.1022.

[12] T.G. Müller, T. Sekiguchi, M. Kaasalainen, M. Abe, S. Hasegawa, Itokawa: The power of ground-based mid-infrared observations, in: A. Milani, G.B. Valsecchi, D. Vokrouhlický (Eds.) Proceedings of the International Astronomical Union Symposium, Cambridge University Press, 2006, pp. 261-266.

[13] F. Zuiani, M. Vasile, A. Gibbings, Evidence-Based Robust Design of Deflection Actions for Near Earth Objects, Celestial Mechanics and Dynamical Astronomy, 114 (2012) pp. 107-136.

[14] R.R. Yager, M. Federizzi, J. Kacprzyk, Advances in the Dempster-Shafer Theory of Evidence, John Wiley \& Sons Inc., Hoboken, 1994.

[15] S. Abe, T. Mukai, N. Hirata, O.S. Barnouin-Jha, A.F. Cheng, H. Demura, R.W. Gaskell, T. Hashimoto, K. Hiraoka, T. Honda, T. Kubota, M. Matsuoka, T. Mizuno, R. Nakamura, D.J. Scheeres, M. Yoshikawa, Mass and Local Topography Measurements of Itokawa by Hayabusa, Science, 312 (2006) p. 1344.

[16] J. Baer, S.R. Chesley, R.D. Matson, Astrometric masses of 26 asteroids and observations on asteroid porosity, The Astronomical Journal, 141 (2011) pp. 143-155.

[17] M. Vasile, C. Colombo, Optimal impact strategies for asteroid deflection, Journal of Guidance, Control, and Dynamics, 31 (2008) pp. 858-872.

[18] J.P. Sanchez, C. Colombo, Impact Hazard Protection Efficiency by a Small Kinetic Impactor, Journal of Spacecraft and Rockets, in press (2012) DOI:10.2514/2511.A32304.

[19] E.J. Öpik, Collision probabilities with the planets and the distribution of interplanetary matter, Proceedings of the Royal Irish Academy. Section A: Mathematical and Physical Sciences, 54 (1951) pp. 165-199.

[20] W.F. Bottke Jr., A. Morbidelli, R. Jedicke, J.-M. Petit, H.F. Levison, P. Michel, T.S. Metcalfe, Debiased Orbital and Absolute Magnitude Distribution of the Near-Earth Objects, Icarus, 156 (2002) pp. 399-433.

[21] Sentinel Mission, in, B612 Foundation, 2012.

[22] H. Agarwal, J.E. Renaud, E.L. Preston, Trust region managed reliability based design optimization using Evidence Theory, in: 44th AIAA/ASME/ASCE/AHS Structures, Structural Dynamics, and Materials Conference, Norfolk, 2003.

[23] K.A. Holsapple, K.R. Housen, Momentum transfer in asteroid impacts. I. Theory and scaling, Icarus, 221 (2012) pp. 875-887. 
[24] C.A. Maddock, J.P. Sanchez, M. Vasile, G. Radice, Comparison of Single and MultiSpacecraft Configurations of NEA deflection by Solar Sublimation, AIP conference Proceedings, 886 (2007) pp. 303-316.

[25] C. Foster, J. Bellerose, D. Mauro, B. Jaroux, Mission concepts and operations for asteroid mitigation involving multiple gravity tractors, Acta Astronautica, (2012) DOI:10.1016/j.actaastro.2012.1010.1010.

[26] D.K. Yeomans, S. Bhaskaran, S.B. Broschart, S.R. Chesley, P.W. Chodas, M.A. Jones, T.H. Sweetser, Near-Earth Object (NEO) analysis of transponder tracking and gravity tractor performance, in, JPL Task Plan No. 82-120022, 2008.

[27] K. Deb, A. Pratap, S. Agarwal, T. Meyarivan, A Fast and Elitist Multiobjective Genetic Algorithm: NSGA-II, IEEE Transactions on Evolutionary Computation, 6 (2002) pp. 182-197.

[28] M. Yoshikawa, A. Fujiwara, J. Kawaguchi, Hayabusa Mission \& Science Team, The nature of asteroid Itokawa revealed by Hayabusa, in: A. Milani, G.B. Valsecchi, D. Vokrouhlický (Eds.) Proceedings of the International Astronomical Union Symposium, Cambridge University Press, 2006, pp. 401-416.

[29] H. Yurimoto, K. Abe, M. Abe, M. Ebihara, A. Fujimura, M. Hashiguchi, K. Hashizume, T.R. Ireland, S. Itoh, J. Katayama, C. Kato, J. Kawaguchi, N. Kawasaki, F. Kitajima, S. Kobayashi, T. Meike, T. Mukai, K. Nagao, T. Nakamura, H. Naraoka, T. Noguchi, R. Okazaki, C. Park, N. Sakamoto, Y. Seto, M. Takei, A. Tsuchiyama, M. Uesugi, S. Wakaki, T. Yada, K. Yamamoto, M. Yoshikawa, M.E. Zolensky, Oxygen Isotopic Compositions of Asteroidal Materials Returned from Itokawa by the Hayabusa Mission, Science, 333 (2011) pp. 1116-1119.

[30] D.T. Britt, G.J. Consolmagno S.J., Stony meteorite porosities and densities: A review of the data through 2001, Meteoritics \& Planetary Science, 38 (2003) pp. 1161-1180.

[31] D.T. Britt, G.J. Consolmagno, Meteorite porosities and densities: A review of trends in the data, in: 35th Lunar and Planetary Science Conference, Houston, 2004.

[32] D.T. Britt, D. Yeomans, K. Housen, G. Consolmagno, Asteroid Density, Porosity, and Structure, in: W.F. Bottke Jr., A. Cellino, P. Paolicchi, R.P. Binzel (Eds.) Asteroids III, University of Arizona Press, Tucson, 2002, pp. 485-500.

[33] A.W. Harris, The H-G asteroid magnitude system: Mean slope parameters, Abstracts of the Lunar and Planetary Science Conference, 20 (1989) p. 375.

[34] B. Mason, The carbonaceous chondrites, Space Science Reviews, 1 (1962) pp. 621-646.

[35] G.J. Flynn, L.B. Moore, W. Klöck, Density and Porosity of Stone Meteorites: Implications for the Density, Porosity, Cratering, and Collisional Disruption of Asteroids, Icarus, 142 (1999) pp. 97-105.

[36] J.A. Wood, Meteorites and the Origin of Planets, McGraw-Hill Book Company, New York, 1968.

[37] E.P. Henderson, S.H. Perry, A discussion of the densities of iron meteorites, 1954, 6 (1954) pp. 221-240.

[38] C.P. Opeil, G.J. Consolmagno, D.T. Britt, The thermal conductivity of meteorites: New measurements and analysis, Icarus, 208 (2010) pp. 449-454.

[39] G.J. Consolmagno S.J., D.T. Britt, The density and porosity of meteorites from the Vatican collection, Meteoritics \& Planetary Science, 33 (1998) pp. 1231-1241.

[40] D. Isbell, F. O'Donnell, J.G. Watson, Deep Space 1 Asteroid Flyby, in, National Aeronautics and Space Administration, 1999.

[41] H. Kuninaka, Round-Trip Deep Space Maneuver of Microwave Discharge Ion Engines onboard HAYABUSA Explorer, in: the 32nd International Electric Propulsion Conference, Wiesbaden, 2011.

[42] C. Gritzner, R. Kahle, Mitigation technologies and their requirements, in: M.J.S. Belton, T.H. Morgan, N.H. Samarasinha, D.K. Yeomans (Eds.) Mitigation of Hazardous Comets and Asteroids, Cambridge University Press, Cambridge, 2004, pp. 167-200.

[43] J.R. Wertz, W.J. Larson, Space Mission Analysis and Design, third edition ed., Microcosm Press, 2003. 